\title{
Proportional Switching in First-in, First-out Networks
}

DOI:

10.1287/opre.2016.1565

\section{Document Version}

Accepted author manuscript

Link to publication record in Manchester Research Explorer

\section{Citation for published version (APA):}

Bramson, M., D'Auria, B., \& Walton, N. (2017). Proportional Switching in First-in, First-out Networks. Operations Research, 65(2), 496-513. https://doi.org/10.1287/opre.2016.1565

\section{Published in:}

Operations Research

\section{Citing this paper}

Please note that where the full-text provided on Manchester Research Explorer is the Author Accepted Manuscript or Proof version this may differ from the final Published version. If citing, it is advised that you check and use the publisher's definitive version.

\section{General rights}

Copyright and moral rights for the publications made accessible in the Research Explorer are retained by the authors and/or other copyright owners and it is a condition of accessing publications that users recognise and abide by the legal requirements associated with these rights.

\section{Takedown policy}

If you believe that this document breaches copyright please refer to the University of Manchester's Takedown Procedures [http://man.ac.uk/04Y6Bo] or contact uml.scholarlycommunications@manchester.ac.uk providing relevant details, so we can investigate your claim.

\section{OPEN ACCESS}




\title{
Submitted to Operations Research manuscript (Please, provide the manuscript number!)
}

Authors are encouraged to submit new papers to INFORMS journals by means of a style file template, which includes the journal title. However, use of a template does not certify that the paper has been accepted for publication in the named journal. INFORMS journal templates are for the exclusive purpose of submitting to an INFORMS journal and should not be used to distribute the papers in print or online or to submit the papers to another publication.

\section{Proportional switching in FIFO networks}

\author{
Maury Bramson \\ School of Mathematics, University of Minnesota, 206 Church St. SE, MN 55455, USA. bramson@math.umn.edu \\ Bernardo D'Auria \\ Departmento de Estadística, Universidad Carlos III de Madrid, Calle Madrid 126, 28903 Getafe, Madrid, Spain. \\ bernardo.dauria@uc3m.es \\ Neil Walton \\ Korteweg-de Vries Institute for Mathematics, University of Amsterdam, Science Park 904, 1098 XH Amsterdam, NL. \\ n.s.walton@uva.nl
}

We consider a family of discrete time multihop switched queueing networks where each packet moves along a fixed route. In this setting, BackPressure is the canonical choice of scheduling policy; this policy has the virtues of possessing a maximal stability region and not requiring explicit knowledge of traffic arrival rates. BackPressure has certain structural weaknesses because implementation requires information about each route, and queueing delays can grow super-linearly with route length. For large networks, where packets over many routes are processed by a queue, or where packets over a route are processed by many queues, these limitations can be prohibitive.

In this article, we introduce a scheduling policy for FIFO networks, the Proportional Scheduler, which is based on the proportional fairness criterion. We show that, like BackPressure, the Proportional Scheduler has a maximal stability region and does not require explicit knowledge of traffic arrival rates. The Proportional Scheduler has the advantage that information about the network's route structure is not required for scheduling, which substantially improves the policy's performance for large networks. For instance, packets can be routed with only next-hop information and new nodes can be added to the network with only knowledge of the scheduling constraints.

Key words: Proportional Scheduler, BackPressure, Kelly networks, bandwidth sharing networks, Massoulié networks, switch networks, proportional fairness.

History:

\section{Introduction}

We consider, in this paper, a family of discrete time multihop switched queueing networks where packets move along fixed routes. Switched networks were first introduced in Tassiulas and Ephremides (1992); this terminology was first employed in Shah and Wischik (2012) for a discrete 
time queueing network whose queues are served simultaneously subject to certain global scheduling constraints and with packets moving along fixed routes. Here, we consider a variant of this model that allows more than one route through each queue; the scheduling constraints and queueing discipline employed here do not depend on the routes of the packets.

Applications of switched networks include wireless ad-hoc networks, Internet routers, call centers with cross trained staff, data centers, and urban road traffic scheduling. For such applications, and in general, maximum stability is a highly desirable feature for the scheduling policy. Roughly stated, for switched networks, a scheduling policy is maximally stable if, for every arrival rate for which there exists a stable policy, the policy stabilizes the network. When the vector of arrival rates is known, one can specify a stable policy by choosing a random schedule whose average service rate at each queue dominates the corresponding arrival rate. However, in practice, explicit knowledge of arrival rates is often not available, particularly when rates may vary. Since the seminal work of Tassiulas and Ephremides (1992), BackPressure has been the canonical maximally stable scheduling policy for multihop switched networks. An important feature of this policy is that, in addition to it being maximally stable, only information on the local state is required for a scheduling decision.

When packets are routed through different network components, policies such as BackPressure require detailed knowledge of this routing. Such policies are similar in nature to the classical single class Jackson queueing networks if one identifies each route with a queue, so that each queue now contains only a single customer type. However, in many applications, this single class interpretation may not be practical because the information required can increase rapidly in the number of queues of the original network. It is also not common practice - for instance, an Internet router will maintain a first-in, first-out (FIFO) queue for each outgoing link rather than a queue for each route, because the number of links to and from a router is orders of magnitude smaller than the number of routes it processes. As a result, in many practical situations, the model simplifies if one reinterprets queues as being multiclass, i.e., permitting different packet classes and hence different routing of packets passing through the queue.

In our setting, queues will be multiclass but will serve packets according to a scheduling policy allocating service among queues that depends only on the number of packets at each queue; the discipline at each queue will be FIFO and packets will all be of unit size. In this setting, we will show that this switching policy, the Proportional Scheduler, is maximally stable.

There are well-known examples of disciplines that are not maximally stable for multiclass queues, for instance, Lu and Kumar (1991), Rybko and Stolyar (1992) and Bramson (1994). (These examples are in both discrete and continuous time.) The examples in Bramson (1994) are for the FIFO discipline, with jobs having unequal mean service requirements. In switch networks, all jobs have equal service requirements and so do not fall within this framework. 
Our policy, the Proportional Scheduler, can be described roughly as follows: for a set of FIFO queues $\mathcal{J}$, a vector of queue lengths $\left(Q_{j}: j \in \mathcal{J}\right) \in \mathbb{Z}_{+}^{|\mathcal{J}|}$, and a convex set of schedules $\left\langle\mathcal{S}>\subset \mathbb{R}^{|\mathcal{J}|}\right.$, the Proportional Scheduler serves packets according to a vector of expected rates, $\boldsymbol{\sigma} \in \mathbb{R}^{|\mathcal{J}|}$, that solves the proportional fair optimization problem

$$
\text { maximize } \quad \sum_{j \in \mathcal{J}} Q_{j} \log \sigma_{j} \quad \text { over } \quad \boldsymbol{\sigma} \in<\mathcal{S}>\text {. }
$$

Packets within a queue are to be served according to a FIFO queueing discipline; once served, a packet goes to the next queue on its route.

We prove that maximal stability holds for this policy by employing a fluid model analysis of our system. This combines the approaches of Massoulié (2007), for bandwidth networks, and Bramson (1996), for FIFO Kelly networks; the latter networks can be viewed as the special case of switched networks where the allocation of service to different queues is fixed irrespective of the state of the system.

We also compare our policy to the BackPressure policy. Unlike BackPressure, the Proportional Scheduler does not require knowledge of the route used by each packet. Thus, routing structure and scheduling decisions are distinct for the Proportional Scheduler policy. As we later discuss in more detail, there are various benefits of the Proportional Scheduler's functional structure: a) rather than distinguishing packets according to their routes (either in the memory of the scheduling algorithm or by physically maintaining different queues), packets can be served at their queue in a first-in, first-out order, b) a packet can be routed knowing only its next hop, rather than knowing its entire route, $\mathrm{c}$ ) when adding new network components, one only needs to know the component's scheduling constraints and not the entire route or class structure of the network to implement the policy, and d) for BackPressure, messages must be sent between queues, which the Proportional Scheduler does not require.

On the other hand, the Proportional Scheduler policy is less general than BackPressure in that the latter model allows for adaptive routing decisions whereas routing for the Proportional Scheduler is fixed.

Another well-known switched network policy is the MaxWeight policy; it is defined by the optimization whose objective function employs the linear factor $\sigma_{j}$, in place of $\log \sigma_{j}$, in (1), i.e.,

$$
\text { maximize } \quad \sum_{j \in \mathcal{J}} Q_{j} \sigma_{j} \quad \text { over } \quad \boldsymbol{\sigma} \in<\mathcal{S}>
$$

As such, it also satisfies the properties a) - d) above. MaxWeight is maximally stable for single hop networks, since it coincides there with the BackPressure policy. 
MaxWeight is not always maximally stable for multihop networks, even when each queue possesses only one class. This is shown in Andrews and Zhang (2003) for fluid models for a weighted version of MaxWeight, after modifying the model slightly by varying the arrival rate of mass; supporting simulations are then given for a queueing network under the standard MaxWeight policy. We briefly consider the maximal stability of MaxWeight in Section 3, but leave a detailed investigation of the policy to future work.

The same question about maximal stability exists for policies with objective functions employing factors other than $\sigma_{j}$ or $\log \sigma_{j}$. As the counterexample in Andrews and Zhang (2003) shows, care needs to be exercised in the choice of the objective function in (1).

\subsection{Relevant Literature}

The main results of this paper combine a number of results, methods, and models from the theory of queueing networks over the last three decades. We review this relevant literature in a roughly chronological order.

A. Classical Queueing Networks: The development of Jackson networks is one of the earliest substantial developments in the theory of stochastic networks. In Jackson networks, a single class of customers is routed probabilistically between queues in the network. Influenced by the Input Theorem of Burke (1956), Jackson (1963) found that the stationary distribution of these networks can be written in product form, meaning that its stationary distribution is the product of simple terms.

Baskett et al. (1975) and Kelly (1975, 1979) broadened this family of queueing networks by permitting queues to be multiclass, and hence allowing more than one route through each queue. Using quasi-reversibility, they showed that, for certain service disciplines including FIFO, the stationary distribution must be of product form. (Quasi-reversibility will also be employed in the context of proportional fairiness in Part D below.)

B. Switched Networks, BackPressure and their Applications: Switched queueing networks and, more specifically, the BackPressure scheduling policies, were first introduced by Tassiulas and Ephremides (1992) as a model of wireless communication. As mentioned above, a switched queueing network is a discrete time queueing network with constraints on which queues can be served simultaneously. The BackPressure policies have proven popular because they maximize a network's stability region while not requiring explicit estimation of traffic arrival rates; for a comprehensive review of the BackPressure policies, see Georgiadis et al. (2006).

The BackPressure policies have been generalized and specialized in numerous directions. In contrast to our work, the defining feature of a BackPressure policy is that the policy minimizes the drift of a Lyapunov function subject to the scheduling constraints of the network. For single hop 
Bramson, D'Auria and Walton: Proportional Switching

Article submitted to Operations Research; manuscript no. (Please, provide the manuscript number!)

networks - where packets are served only once before departing the network - the BackPressure policy is often referred to as the MaxWeight policy. As a model of Internet protocol routers, McKeown et al. (1999) applied this paradigm to the example of input-queued switches. Andrews et al. (2004) consider power functions when defining their MaxWeight Lyapunov function and thus generalized the set of MaxWeight/BackPressure policies. Additional extensions are considered by Meyn (2009) and Eryilmaz et al. (2005) and further generalizations to cone polices are considered by Armony and Bambos (2003). In different stochastic senses, BackPressure and MaxWeight can be shown to optimize certain workload functions: in heavy traffic, see Stolyar (2004); in large deviations, see Venkataramanan and Lin (2007); in overload, see Shah and Wischik (2011). Further, heavy tailed arrivals are analyzed by Jagannathan et al. (2011); delay in the presence of heavy tailed traffic are considered in Markakis et al. (2014).

There are numerous application areas associated with switched networks. For these areas, BackPressure is often the canonical choice. Applications include wireless ad-hoc networks, in Tassiulas and Ephremides (1992), Internet routers, in McKeown et al. (1999), call centers with cross trained staff, in Mandelbaum and Stolyar (2004), data centers, in Shah and Wischik (2011), urban road traffic scheduling, in Varaiya (2013), and stochastic processing networks, which would include numerous manufacturing and general processing settings, in Dai and Lin (2005).

C. Instability, Fluid Stability and Stability of Queueing Networks: Most classical queueing networks are positive recurrent when the network is subcritical. By positive recurrent, we mean that there exists a state (which, in our setting, will be the state with no packets) that is positive recurrent and that this state is visited with probability 1 starting from any other state; this is the reduction to the countable state space setting of positive Harris recurrence, which is the standard definition in the general state space setting. By subcritical, we mean that each network resource experiences a load that is strictly less than the resource's capacity. It had been thought that subcritical networks were always positive recurrent under a work conserving policy. However, a series of examples constructed in the mid-nineties showed that this is not the case. For instance, see $\mathrm{Lu}$ and Kumar (1991), Rybko and Stolyar (1992) and Bramson (1994).

This led to new approaches for determining the stability region for queueing networks. In particular, Rybko and Stolyar (1992) and Dai (1995) developed a fluid model approach where the stability of a queueing network can be determined from that of an associated fluid model. This theory is surveyed in by Bramson (2008). A fluid analysis of multiclass FIFO queueing networks was first given by Bramson (1996). Our fluid analysis uses a similar approach.

Recent work of Dieker and Shin (2013) further considers the issue of finding natural scheduling policies that lead to stability whenever each server is nominally underloaded. Similar to adversarial queueing frameworks, e.g., Borodin et al. (2001), stability is achieved by prioritizing queue service 
according to a least-routed-first-priority discipline. This differs from the approach taken in this paper where the service discipline does not use the routing structure of the network to achieve maximum stability. Further recent work of Ji et al. (2013) considers stability results for switched networks where packets are queued per-link rather than per-route, as is typically applied for BackPressure. Here stability is achieved by running an appropriate queueing system in the memory of the algorithm. By estimating queue sizes and loads in this way, stability is achievable for switched networks as long as routes do not form a loop. Once again this differs from the approach of this paper, where routes are general and only current queue size information is required to execute the scheduling policy. Finally, Ying et al. (2011) consider a modification of the BackPressure policy to lessen the effect of per-route queueing. Queues are grouped into predetermined clusters, with the standard BackPressure policy applied within each cluster. A judicious choice of clusters will reduce the amount of required memory. Clusters are centrally determined and, as with BackPressure, per-link queueing is not employed and information on the queue size must be continuously exchanged along queues between the source and destination.

D. Massoulié Networks, Proportional Fairness and Quasi-Reversibility: A further class of Internet models was introduced in Massoulié and Roberts (1999) and Massoulie and Roberts (2000) in a processor-sharing framework where resources are shared subject to constraints on these resources. Similar to MaxWeight and BackPressure, these policies are often defined by an optimization that is maximally stable. (Unlike BackPressure, the construction does not minimize the instantaneous drift of a Lyapunov function.) Stability proofs for these systems can be found in Bonald and Massoulié (2001), Ye et al. (2005), Gromoll and Williams (2009) and Paganini et al. (2012). Due to their proliferation to different areas, these models have taken various names, such as bandwidth sharing networks, stochastic flow level models, and resource sharing networks; here, we refer to these networks as Massoulié networks. See Harrison et al. (2014) for a recent discussion of the benefits and varied applications of this resource sharing paradigm.

In this paper, we allocate resources according to the proportional fair optimization, which was first introduced by Kelly (1997). Proportional fairness has been used in the allocation of bandwidth in modern 3G telephone networks, see, e.g., Viswanath et al. (2002) and Kushner and Whiting (2004). The stability of proportional fairness in Massoulié networks was first shown by De Veciana et al. (1999); an important generalization of this stability analysis is given in Massoulié (2007). Further progress on the stability and large deviations behavior of proportional fairness can be found in Jonckheere and López (2014). Heavy traffic analysis of proportional fair policies can be found in Kang et al. (2009), Ye and Yao (2012), and Vlasiou et al. (2014). Stolyar (2004) has investigated resource pooling for MaxWeight policies Kang et al (2007) Kang et al (2009) Kelly 
et al. (2009) and Ye and Yao (2012) discussed product form resource pooling properties associated with the proportional fairness in heavy traffic and large deviations regimes.

Influenced by Whittle (1985), the quasi-reversibility and insensitivity property in Massoulié networks was studied by Bonald and Proutière 2002, 2003, 2004) and Zachary (2007). For connections between proportional fairness and the queueing networks of Kelly (1975) and Baskett et al. (1975), see, e.g., Schweitzer (1979), Kelly (1989), Massoulié and Roberts (1999), Walton (2009), and Anselmi et al. (2013). A short, general description of the relationship between proportional fairness, maximum stability and quasi-reversibility can also be found in Walton (2011). As we discuss later, the Lyapunov function in Massoulié (2007) is relevant to our analysis.

E. Resource Sharing in Switched Networks: The sharing of network resources is a key property of the proportional fair optimization. Other resource sharing policies exist, e.g., the weighted $\alpha$-fair policies of Mo and Walrand (2000). Only recently, authors have begun to consider these policies in the context of switched networks; to the best of our knowledge, application of $\alpha$-fairness to switched networks was first made by Shah and Wischik (2011) and Zhong (2012). (Added in revision: Li and Srikant (2016) has shown stability of a per-link scheduling policy for multihop networks.)

\subsection{Organization}

The remainder of the paper is organized as follows. In Section 2, we define a family of FIFO switched networks and the Proportional Scheduler. The main result of the paper, Theorem 1, states that the corresponding network is positive recurrent for all subcritical arrival rates. In Section 3, we discuss the properties of the Proportional Scheduler in comparison to BackPressure; the section is not needed to understand Theorem 1, but it is important in order to understand its consequences. In Section 4, we prove Theorem 1. We begin by characterizing the fluid model that is associated with the Proportional Scheduler and then, in 20 22 and Proposition 1, define a Lyapunov function for the fluid model and calculate its derivative. This is applied, in Theorem 2, to prove fluid stability, from which we conclude, in Proposition 3, that the corresponding stochastic network is positive recurrent. Proposition 3 and certain other steps in the proof of Theorem 1 will be proved in the appendix.

\section{FIFO Network Model, Proportional Scheduler, and Main Result}

\subsection{Network and Scheduling Set Notation}

Let $\mathcal{J}$ be a finite set of queues, with cardinality $|\mathcal{J}|$ and indexed by $j$. A schedule is a vector $\sigma=\left(\sigma_{j}: j \in \mathcal{J}\right) \in \mathbb{Z}_{+}^{|\mathcal{J}|}$, where $\mathbb{Z}_{+}^{|\mathcal{J}|}$ denotes the non-negative integers. We will denote by $\mathcal{S}$ a finite set of schedules satisfying: (1) If $\boldsymbol{\sigma} \in \mathcal{S}$, then $\tilde{\boldsymbol{\sigma}} \in \mathcal{S}$ for each $\tilde{\boldsymbol{\sigma}} \leq \boldsymbol{\sigma}$ (with vector inequalities $\tilde{\boldsymbol{\sigma}} \leq \boldsymbol{\sigma}$ being interpreted componentwise, i.e., $\tilde{\sigma}_{j} \leq \sigma_{j}$ for all $\left.j \in \mathcal{J}\right)$. Note that the vector of all zeros 
belongs to $\mathcal{S}$. (2) For each $j \in \mathcal{J}$, there exists some schedule $\boldsymbol{\sigma} \in \mathcal{S}$ such that $\sigma_{j}>0$. For a vector $\mathbf{Q}=\left(Q_{j}: j \in \mathcal{J}\right) \in \mathbb{Z}_{+}^{\mathcal{J}}$, we denote by $\mathcal{S}_{Q}$ the schedules in $\mathcal{S}$ with $\sigma_{j} \leq Q_{j}$ for $j \in \mathcal{J}$, and denote by $\sigma_{\max }:=\max \left\{\sigma_{j}: j \in \mathcal{J}, \boldsymbol{\sigma} \in \mathcal{S}\right\}$ the maximum component in the set of schedules. We define $<\mathcal{S}>$ to be the convex combination of points in $\mathcal{S}$ and assume that $\langle\mathcal{S}\rangle$ has non-empty interior. The subcritical region $\mathcal{C}$ of this network is the interior of $\langle\mathcal{S}\rangle$. (In the setting of multiclass queueing networks with a fixed service rate at each queue, $\langle\mathcal{S}\rangle$ becomes a rectangle with faces parallel to the axes.)

Each packet in the network is assumed to belong to a class at a given time $t$. The notation of a packet's class will be used to uniquely identify the route of the packet, the queue it is at, and the stage along its route. We denote by $\mathcal{K}$ the set of classes of packets in the network. A route through the network is a vector of classes $r=\left(k_{i}^{r}: i=1, \ldots,|r|\right) \in \mathcal{K}^{|r|}$, with size $|r| \in \mathbb{N}$. Each class is assumed to occur along a unique route, with the class occurring exactly once along its route; $\mathcal{R}$ denotes the set of routes through this network.

With each class $k \in \mathcal{K}$, we associate a unique route denoted $r(k)$ and a unique queue $j(k)$. For notational convenience, we add an additional "outside" class denoted by $\omega$ : For each class $k \in \mathcal{K}$, we let the function $b(k) \in \mathcal{K} \cup\{\omega\}$ denote the class before class $k$ on route $r$; if $k$ is the first class on a route, we then set $b(k)=\omega$. Similarly, the function $n(k) \in \mathcal{K} \cup\{\omega\}$ will denote the next class on route $r$. If $k \in \mathcal{K}$ is the last class on a route, then $n(k)=\omega$.

For a given route $r \in \mathcal{R}$, we define the input class $i(r) \in \mathcal{K}$ to be the first class on route $r$, i.e., $i(r)=k_{1}^{r}$, and the output class $o(r) \in \mathcal{K}$ to be the last class on route $r$, i.e., $o(r)=k_{|r|}^{r}$. The subsets of input and output classes are denoted by $\mathcal{K}^{i}$ and $\mathcal{K}^{o}$, respectively. For notational convenience, we write $k \in j$ to indicate that class $k$ is at queue $j$, i.e., $j(k)=j$, and $k \in r$ to indicate that class $k$ occurs on route $r$, i.e., $r(k)=r$. Unless stated otherwise, $|\cdot|$ denotes the $L^{1}$ norm.

\subsection{Network Quantities and Equations}

Our principal objects of interest are discrete time FIFO networks and the Proportional Scheduler, which are described here and in the next subsection. We begin by introducing the state primitives for first-in, first-out (FIFO) networks having time index $t \in \mathbb{Z}_{+}$. Analogous primitives and equations will be employed in Section 4 for the corresponding fluid model, where time will instead be continuous and the model will be both continuous and deterministic.

Throughout the paper, the indices $j, k, r$ will be used to refer to, respectively, queues $\mathcal{J}$, classes $\mathcal{K}$ and routes $\mathcal{R}$. Regardless of the index $x=j, k, r$, we will denote by $A_{x}(t)$ the cumulative number of arrivals by time $t$, by $D_{x}(t)$ the cumulative departures by time $t$, and by $Q_{x}(t)$ the queue size at time $t$. For example, $A_{j}(t)$ is the total number of arrivals at queue $j$ by time $t, D_{k}(t)$ is the total number of packets that have departed from class $k$, and $Q_{r}(t)$ is the number of packets that have 
arrived at but not departed from route $r$. For each $k \in \mathcal{K}$ and $s \in \mathbb{N}$, the function $\Gamma_{k}(s)$ denotes the number of packets of class $k$ that will be served after $s$ packets are served from queue $j(k)$.

The processes $A_{x}(t), D_{x}(t)$ and $\Gamma_{k}(t)$ are non-negative and non-decreasing, with $A_{x}(0)=D_{x}(0)=$ $\Gamma_{k}(0)=0$ for $x \in \mathcal{J} \cup \mathcal{K} \cup \mathcal{R}$ and $k \in \mathcal{K}$. The queue size process $Q_{x}(t)$ is non-negative for $x \in$ $\mathcal{J} \cup \mathcal{K} \cup \mathcal{R}$. Given these natural conditions, the following fundamental equations hold for a FIFO switched network.

$$
\begin{aligned}
& Q_{x}(t)=Q_{x}(0)+A_{x}(t)-D_{x}(t), \\
& \sum_{k \in j} \Gamma_{k}(t)=t, \\
& \left(\frac{D_{j}(t)-D_{j}(s)}{t-s}: j \in \mathcal{J}\right) \in<\mathcal{S}>\text { for } t>s, \\
& D_{k}(t)=\Gamma_{k}\left(D_{j}(t)\right), \\
& A_{k}(t)+Q_{k}(0)=\Gamma_{k}\left(A_{j(k)}(t)+Q_{j(k)}(0)\right), \\
& A_{k}(t)=D_{b(k)}(t),
\end{aligned}
$$

where $x \in \mathcal{J} \cup \mathcal{K} \cup \mathcal{R}, j \in \mathcal{J}, k \in \mathcal{K}$ and $r \in \mathcal{R}$ for (347), and $k \in \mathcal{K} \backslash \mathcal{K}^{i}$ in (8). Finally, we define the arrivals/departures for routes by

$$
A_{r}(t)=A_{i(r)}(t), \quad D_{r}(t)=D_{o(r)}(t)
$$

The above equations (3,8) can be interpreted as follows: (3) is standard, (4) states that the $t$ th packet served from queue $j$ is from class $k \in j$, and (5) states that the departure process must be achievable within the constraints of the network scheduling. In $(6), \Gamma_{k}\left(D_{j}(t)\right)$ is the number of class $k$ packets served after $D_{j}(t)$ units of service; (7) states that all of the packets that were originally at class $k$ or arrived there by time $t$ will have been served after $A_{j}(t)+Q_{j}(0)$ packets have been served at $j$ and so, if the $A_{j}(t)$ th packet arrival is of class $k$, then the $\left(A_{j}(t)+Q_{j}(0)\right)$ th packet departure is also of class $k$, i.e., the queueing discipline is FIFO; (8) gives the routing between classes. Further relationships can also be deduced from the above equations. For instance, (4) and (7) imply $A_{j}(t)=\sum_{k \in j} A_{k}(t)$ and $D_{j}(t)=\sum_{k \in j} D_{k}(t)$, and (3), (9) and (8) imply $Q_{r}(t)=\sum_{k \in r} Q_{k}(t)$.

We remark that, when the service rates $\sigma_{j}$ are constant, the FIFO property given by (7) is equivalent to the FIFO property (2.5) in Bramson (1996). The FIFO condition there is given in terms of the workload at the queue; such an interpretation of workload does not immediately transfer to our setting, since service rates can vary. Also note that (7) does not restrict the order in which packets that have arrived simultaneously at a queue are served; we shall allow any such order. 
In addition to equations (3,8), we also assume that the number of arrivals at each route $r$ over different times is i.i.d. with mean $a_{r} \in(0, \infty)$, and that the arrivals at different routes occur independently. For each class $k \in r$, we set $a_{k}=a_{r}$, and for each queue $j \in \mathcal{J}$, we set $a_{j}=\sum_{k \in j} a_{k}$, with $\boldsymbol{a}=\left(a_{j}: j \in \mathcal{J}\right)$. We assume that the time required for the service of each packet is deterministic and equal to 1 . With this in mind, we say that the mean arrival vector $\boldsymbol{a}$ is subcritical for a given network when $\boldsymbol{a} \in \mathcal{C}$; observe that, since $\mathcal{C}$ is open, this implies that $(1+\epsilon) \boldsymbol{a} \in \mathcal{C}$ for some $\epsilon>0$. This provides a natural extension to the definition of subcriticality used for multiclass queueing networks (with a fixed allocation of service for each queue).

We note that for a network with the FIFO property, one needs to specify the initial state of the system by including $\Gamma_{k}(s)$, for $0 \leq s \leq Q_{j}(0)$, in order to uniquely specify the corresponding Markov process. As mentioned above, one also needs to define a tie-breaking rule when two or more packets from different classes arrive at a queue at the same time, for which we specify in the functions $\Gamma_{k}$ which packet arrived "first".

\subsection{Proportional Scheduler}

A scheduling policy is a sequence of schedules $\boldsymbol{\pi}(t) \in \mathcal{S}$ with $\boldsymbol{\pi}(t) \leq \mathbf{Q}(t)$ component-wise that determines the service of packets at each queue, i.e., $\mathbf{D}(t)-\mathbf{D}(t-1)=\boldsymbol{\pi}(t)$. The Proportional Scheduler, which is the main focus of this paper, is defined as follows. For $\mathbf{Q}=\left(Q_{j}: j \in \mathcal{J}\right) \in \mathbb{Z}_{+}^{|\mathcal{J}|}$, let $\boldsymbol{\sigma}(\mathbf{Q})=\left(\sigma_{j}(\mathbf{Q}): j \in \mathcal{J}\right) \in<\mathcal{S}>$ be a solution to the following optimization problem:

$$
\text { maximize } \quad \sum_{j \in \mathcal{J}} Q_{j} \log \sigma_{j} \quad \text { over } \quad \boldsymbol{\sigma} \in<\mathcal{S}_{\mathbf{Q}}>\text {; }
$$

when $Q_{j}=0$, set $\sigma_{j}(\mathbf{Q})=0$, with the convention that $0 \log 0=0$. (Note that $\mathbf{Q} \mapsto \boldsymbol{\sigma}(\mathbf{Q})$ is invariant under scalar multiplication, i.e., $\boldsymbol{\sigma}_{j}(c \mathbf{Q})=\boldsymbol{\sigma}_{j}(\mathbf{Q})$ for $c>0$.) The solution to this optimization need not belong to the set of schedules $\mathcal{S}$. However, since $\boldsymbol{\sigma} \in\left\langle\mathcal{S}_{\mathbf{Q}}>, \boldsymbol{\sigma}\right.$ can be expressed as a convex combination of points in $\mathcal{S}_{\mathbf{Q}}$, i.e., there exists random $\boldsymbol{\pi}(\mathbf{Q})=\left(\pi_{j}(\mathbf{Q}): j \in \mathcal{J}\right)$, with support in $\mathcal{S}$ and such that, for $j \in \mathcal{J}$,

$$
\mathbb{E} \pi_{j}(\mathbf{Q})=\sigma_{j}(\mathbf{Q})
$$

The Proportional Scheduler is defined to be any policy having the sequence of schedules $\boldsymbol{\pi}(t)$, where

$$
\boldsymbol{\sigma}(t)=\arg \max \sum_{j \in \mathcal{J}} Q_{j}(t-1) \log \sigma_{j} \quad \text { over } \quad \boldsymbol{\sigma} \in<\mathcal{S}_{\mathbf{Q}(t-1)}>
$$

and $\boldsymbol{\pi}(t)$ satisfies the analog of $11 \mathrm{a}$ ). (The only sources of randomness in the paper are the choice of $\boldsymbol{\pi}$ in (11a) and the random exogenenous arrivals $A_{r}(t)$ in $(9)$.)

Note that $\boldsymbol{\sigma}(t)$ is uniquely defined because of the strict concavity of the objective function in (10) and because $\sigma_{j}(t)=0$ when $Q_{j}(t)=0$, although $\boldsymbol{\pi}(t)$ need not be uniquely defined (depending 
Bramson, D'Auria and Walton: Proportional Switching

Article submitted to Operations Research; manuscript no. (Please, provide the manuscript number!)

on $\mathcal{S}$ ). Also note that the Proportional Scheduler (and MaxWeight) policies reduce to the FIFO policy of a multiclass queueing network, having a fixed service rate at each queue, when $<\mathcal{S}>$ is a rectangle with faces parallel to the axes.

We will refer to any discrete time Markov chain satisfying the FIFO switched network equations (3-8), along with the policy in (11) and the arrival and service assumptions in the next to last paragraph of Subsection 2.2, as a proportional switched network. The state space of the Markov chain is assumed to be that induced by the number of packets at each queue together with their respective ordering within the queue. (With a slight abuse of terminology, we blur here the distinction between the Markov chain and the underlying switched network.) Recall that positive recurrent means that there exists a state (here, the state with no packets) that is positive recurrent and that this state is visited with probability 1 starting from any other state. (This is the reduction to the countable state space setting of positive Harris recurrence from the general state space setting.)

\subsection{Main Result}

A standard fact is that, under any policy, a queueing network cannot be positive recurrent when the vector of arrival rates $\boldsymbol{a}$ lies outside the network's subcritical region $\mathcal{C}$. It follows that $\mathcal{C}$ is the network's greatest possible stability region. The main result in this paper is the following converse, which shows that the Proportional Scheduler achieves its greatest possible stability region.

TheOREM 1. Suppose that the vector of arrival rates $\boldsymbol{a}=\left(a_{j}: j \in \mathcal{J}\right) \in \mathcal{C}$. Then the corresponding proportional switched network is positive recurrent.

Although this paper considers only proportional switched networks in the discrete time context, we remark that the analogous model can be defined in continuous time, with packets instead requiring an exponential amount of service and the policy being updated immediately after each change in the state of the network. The proof of Theorem 1 relies on the application of fluid models to the proportional switched network and is insensitive to whether time is discrete or continuous, and so will carry over to the continuous time setting. As in the context of FIFO multiclass queueing networks, the main requirement for proportional switched networks on their service times is that their means be the same for different classes at the same queue, although the means may differ among different queues. In our setting, the assumption that service times are all of unit size at different queues is due to the setting of switched networks, rather than intrinsic mathematical requirements for Theorem 1

As is the case for proportional switched networks, the well-known BackPressure networks are also positive recurrent for all subcritical arrival rates. In the next section, we will compare the implementability of the two models. 


\section{Comparison with the BackPressure}

The BackPressure policy is currently the canonical policy for scheduling in multi-hop switched networks. Here, we present examples to illustrate practical differences between this policy and the Proportional Scheduler. This section is not required for the remainder of the paper.

\subsection{Definition of BackPressure}

We define and briefly describe the BackPressure policy using the notation introduced in Section 2 . with the reader being referred to Tassiulas and Ephremides (1992) for a more detailed description of the policy and its properties. The network is defined there in terms of a directed graph, but the reader can easily check that the definition we give below is equivalent; this alternative format is employed to avoid unnecessary complications. (Note that queues $j \in \mathcal{J}$ are referred as links in Tassiulas and Ephremides (1992), and classes are referred to as queues there.)

For a given buffer size distribution $\mathbf{Q}=\left(Q_{k}: k \in \mathcal{K}\right)$, the BackPressure policy can be defined as follows:

1. For each queue $j \in \mathcal{J}$, introduce the weights

$$
w_{j}(\mathbf{Q})=\max _{k \in j}\left\{Q_{k}-Q_{n(k)}\right\}
$$

with $Q_{n(k)}=0$ for $n(k)=\omega$. Denote by $k_{j}^{*}(\mathbf{Q})$ one of the classes where the above maximization is achieved.

2. Over the set of schedules $\mathcal{S}$, solve the optimization

$$
\sigma^{*}(\mathbf{Q}) \in \underset{\sigma \in \mathcal{S}}{\arg \max } \sum_{j \in \mathcal{J}} \sigma_{j} w_{j}(\mathbf{Q}) .
$$

3. When $w_{j}(\mathbf{Q})>0$, schedule $\sigma_{j}^{*}(\mathbf{Q})$ packets from class $k_{j}^{*}(\mathbf{Q})$ at queue $j \in \mathcal{J}$ and no packets from any of the other classes at $j$ during the next time increment; when $w_{j}(\mathbf{Q}) \leq 0$, do not schedule any packets at the queue.

Assuming that the number of arrivals of packets at each route over different times is i.i.d. and the arrivals at different routes occur independently, then the BackPressure policy is positive recurrent whenever the arrival rates are subcritical. This is shown by employing a quadratic Lyapunov function; the BackPressure policy, in fact, maximizes the negative drift of the Lyapunov function.

\subsection{Complexity of the Proportional Scheduler versus that of BackPressure}

The robust stability of the BackPressure policy is a compelling feature. However, a crucial disadvantage of the BackPressure approach is that it leads to a priority policy that requires explicit information about the classes and routes throughout the network; in many practical circumstances, the compilation of such information is not feasible. 


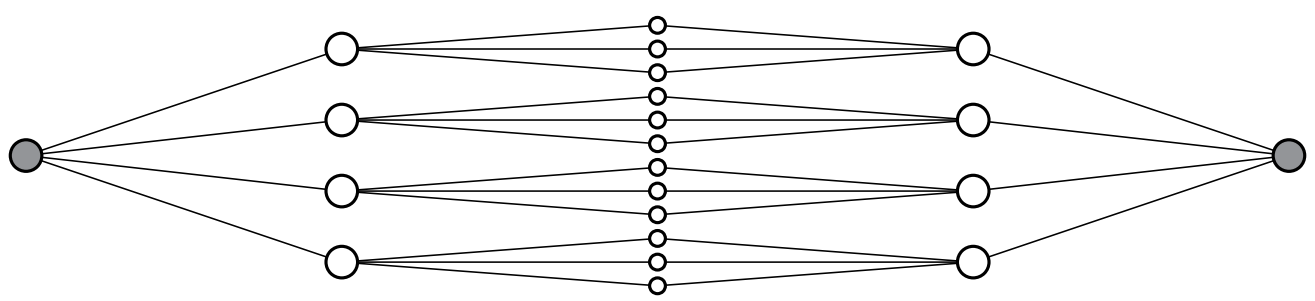

Figure 1 A network of degree $d=4$ and diameter $D=4$.

Consider, for example, the network of diameter $D \in 2 \mathbb{Z}_{+}$that is constructed by joining the leaf nodes of two trees of diameter $D / 2$ each, where each non-leaf node has degree $d$ - the case of a network with degree $d=4$ and diameter $D=4$ is given in Figure 1 . The routes in this network are the paths between the two root nodes, both from left-to-right and from right-to-left. (The two root nodes are colored grey in Figure 1.) No scheduling constraints on the nodes are assumed, and packets require unit service at each node, with each packet visiting each node along its path. In terms of our previous terminology, nodes here will correspond to queues, and each distinctive path emanating from a given node will correspond to a different class at that node. (Paths that merge at a node and remain the same thereafter will correspond to the same class there.)

For scheduling at each node, the BackPressure policy employs computations along each of these classes. Focusing on one of the two root nodes for concreteness, and denoting by $b(d, D)$ the number of classes at the node, it is easy to check that

$$
b(d, D)=d(d-1)^{D / 2-1}+1
$$

which grows rapidly with respect to $d$ and $D$. If implemented in this and other settings with large networks, the BackPressure policy will present serious memory problems. Moreover, the relevant routing information may not be available.

In contrast to this, for the Proportional Scheduler policy, each node requires only knowledge of the number of packets destined for its $d$ adjacent nodes (and not the number of packets in each of its classes). This amount of information is far less than that required by the BackPressure policy in (14); this quantity does not increase as the network size increases, since it depends only on the local structure of the network and not, for example, on the routing or final destination of each packet.

Although the network in Figure 1 is used for reasons of exposition, the Proportional Scheduler is far closer in nature to the next-hop routing used in modern IP routers on the Internet, or that might be used in a wireless ad-hoc network. We note, for instance, that modern Internet routers maintain tens of FIFO queues that aggregate tens of thousands of flows (route classes) (McKeown (1999), Appenzeller et al. (2004)). Furthermore, when these components are connected together to 
form a network, queue state information is not explicitly changed. (We discuss this in Subsection 3.5.)

\subsection{Simulations for a Modification of the Network of Figure 1}

In Figure 2, we provide simulations for the average total queue size for a modification of the network in Figure 1. Specifically, rather than immediately exiting from the root node at the end of its route, a packet returns to this node a geometric number of times, each time with probability $1-\delta, \delta \in(0,1]$, before finally exiting from the network. (We could instead stipulate that the packet returns to the node a fixed number of times before exiting.)

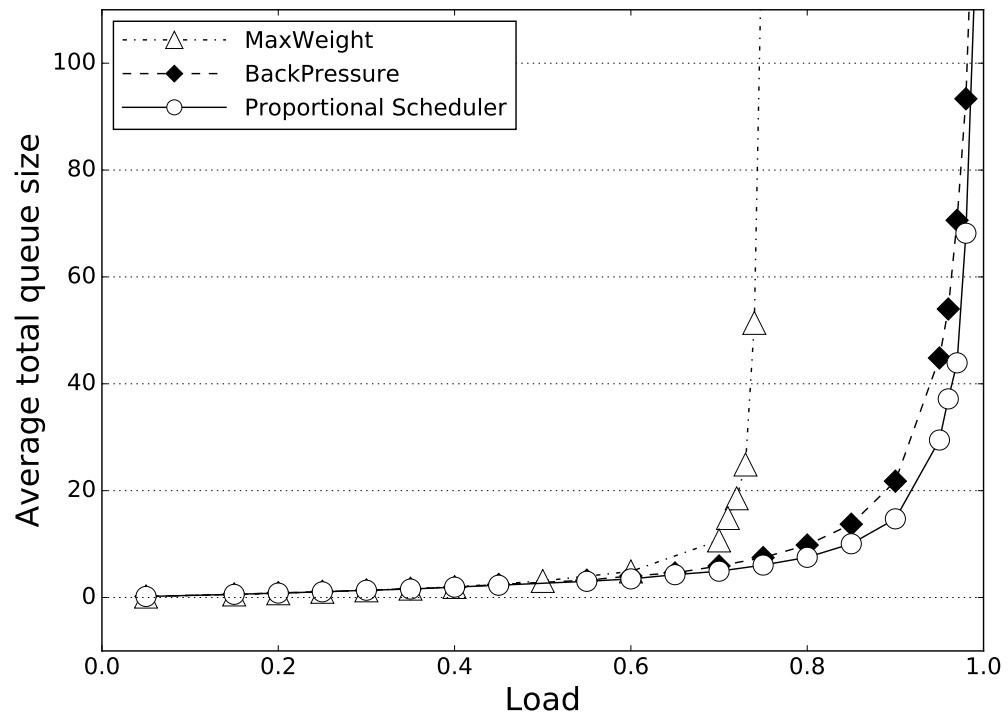

Figure 2 Total queue size of MaxWeight, BackPressure, Proportional Scheduler under a range of loads.

For this modified network, the load at a root node is $a(1+1 / \delta)$ if $a$ is the external arrival rate at each root node, and so $a(1+1 / \delta)<1$ is an obvious necessary condition for positive recurrence of the network, whereas, for both BackPressure and the Proportional Scheduler, the condition is sufficient by Tassiulas and Ephremides (1992) and Theorem 1. For parameters $(D, d, \delta)=(4,4,0.4142)$, we simulated in Figure 2 three policies, BackPressure, the Proportional Scheduler, and MaxWeight, over a range of loads. Our simulations indicate that, as expected, BackPressure and the Proportional Scheduler are maximally stable, whereas, consistent with the examples of input-queued switches in Andrews and Zhang (2003), MaxWeight is not.

We note that the MaxWeight has a stability region that is approximately $75 \%$ of the total capacity. ( $\delta=.4142$, which is about $\sqrt{2}-1$, was chosen so that value would not be close to 1 .) In 
fact, MaxWeight will lose up to $50 \%$ of the capacity for networks of this type; as mentioned in the introduction, more detail on MaxWeight will be provided in a future work. We also note that, under all loads measured in Figure 2, the average total queue size for the Proportional Scheduler was found to be somewhat smaller than that for BackPressure. We will discuss this relationship for another family of networks in Section 3.4

\subsection{Delay on Long Routes}

As illustrated in Subsection 3.2, the complexity of the BackPressure policy is an issue when there are many routes. Other complications might also arise, even when all packets have the same route. Consider, for example, the network in Figure 3, which is a special case of the linear networks considered by Bui et al. (2011) (in Theorem 2) and Stolyar (2011), for the BackPressure policy.

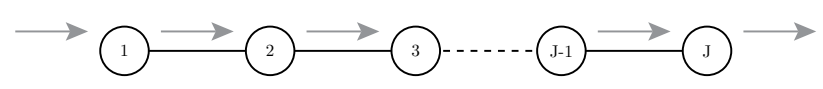

Figure 3 (Above) A linear network with $J$ links. Each packet must pass through each link.

Figure 4 (Right) Queue lengths for the linear network with $J=20$ links.

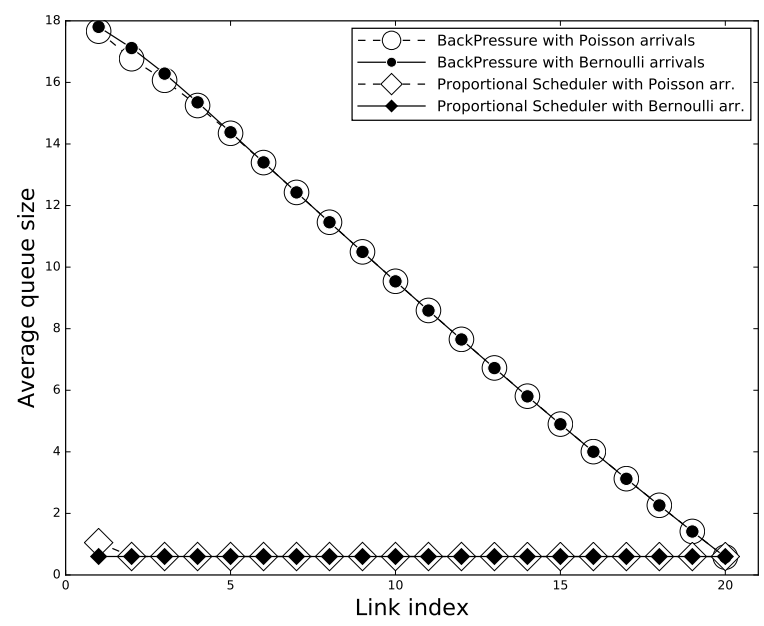

In this example, there are $J \geq 2$ links in series, with packets entering at link 1 and being sequentially processed through links $1,2, \ldots, J$; there are no constraints preventing simultaneous service at different links and, once served, a packet moves to the next link along its route. Arrivals at queue 1 occur according to a Bernoulli process, with mean parameter $a>0$, and one packet is served per unit time at each nonempty queue. Bui et al. (2011) and Stolyar (2011) showed that, if the network is subcritically loaded with $1 / 2<a<1$, then, under the BackPressure policy, the sum of the expected queue sizes in equilibrium grows quadratically in $J$, that is,

$$
\sum_{j=1}^{J} \mathbb{E}\left[Q_{j}\right] \geq c J^{2}
$$

for some constant $c>0$ not depending on $J$. Since one unit of time is required to serve each packet at a queue, it is immediate from (15) that the flow delay for packets satisfies the same lower bound. 
The basic idea behind the demonstration of (15) is that BackPressure only serves a packet on the $j$ th link when $Q_{j}-Q_{j+1}>0$, which will occur in equilibrium with probability a (since the average arrival and departure rates are equal), whereas, in equilibrium, $Q_{j}-Q_{j+1} \geq-1$ must always hold (since service from queue $j$ occurs only when $Q_{j}-Q_{j+1}>0$ ). Since $a>1 / 2$, there will be linear growth in the expected size of successive queues from the last queue $Q_{J}$ to the first queue $Q_{1}$, which implies the quadratic growth in $J$ of the sum of the expected queue sizes. In general, when routing is more involved, one should expect the sum of the queue sizes in equilibrium to grow quadratically with route length for routes with close to critical arrival rates since, as before, BackPressure will not serve links with a negative queue size differential. (For details, see Bui et al. (2011) or Stolyar (2011).)

The Proportional Scheduler (as well as any other work conserving scheduler) will exhibit completely different behavior for this linear network since there are no constraints preventing simultaneous service at different links. In equilibrium, queue 1 will have either 1 or 0 packets corresponding to whether or not an arrival occurred in the last time slot. Therefore, the output of a queue is a Bernoulli process that is independent of the current state of the queue. Arguing inductively, this implies that the queue sizes at different queues are independent, and hence that the sum of the queue sizes for the network is binomially distributed with parameters $J$ and $a$. Consequently, under the Proportional Scheduler policy, the sum of the expected value of the queue sizes in equilibrium grows only linearly in $J$, with

$$
\sum_{j=1}^{J} \mathbb{E}\left[Q_{j}\right]=a J .
$$

The above reasoning that was employed for 16 is an elementary variant of Burke's Output Theorem (as in, e.g., Burke (1956) or Hsu and Burke (1976)).

In Figure 4, we have simulated the linear network in Figure 3, with $J=20$ queues, and assuming an arrival rate of 0.6 of packets into the network and a maximal service rate of 1 at each node. In addition to the case of Bernoulli input discussed above, Poisson input is also simulated for both BackPressure and the Proportional Scheduler. As expected from the above discussion, for BackPressure, the average queue size is proportional to the number of hops from the terminal destination while, for the Proportional Scheduler, the average queue size is constant; in neither of these cases, is the average queue size sensitive to the distribution of the input except for the first few links, as illustrated by the almost perfect overlay of the pairs of graphs. It follows that, as the length of the linear network grows, queueing delay grows quadratically for BackPressure and linearly for the Proportional Scheduler. (Similar observations were made in the simulations conducted by Bui et al. (2011).) 
Bramson, D'Auria and Walton: Proportional Switching

Article submitted to Operations Research; manuscript no. (Please, provide the manuscript number!)

\subsection{Decomposition}

Decomposition is essential to the decentralized implementation of a policy. A property of BackPressure optimization is that, once queue length comparisons have been made between links (see (12), the optimization can be decomposed. In particular, if the scheduling of one subset of queues $\mathcal{J}_{1}$ does not effect the scheduling choice of a complementary subset $\mathcal{J}_{2}$ (i.e., $\mathcal{S}=\mathcal{S}_{1} \times \mathcal{S}_{2}$ ), then the BackPressure optimization can be decomposed as

$$
\max _{\sigma \in \mathcal{S}}\left\{\sum_{j \in \mathcal{J}} \sigma_{j} w_{j}(\mathbf{Q})\right\}=\max _{\sigma \in \mathcal{S}_{1}}\left\{\sum_{j \in \mathcal{J}_{1}} \sigma_{j} w_{j}(\mathbf{Q})\right\}+\max _{\sigma \in \mathcal{S}_{2}}\left\{\sum_{j \in \mathcal{J}_{2}} \sigma_{j} w_{j}(\mathbf{Q})\right\}
$$

The sub-problems involving the two optimizations on the right can then be solved independently, leading to a decomposed implementation of the policy. We remark, however, that the above optimization may not completely decompose since the weight calculations $w_{j}(Q)$ typically require comparisons with the sizes of upstream queues, and hence some information exchange will occur between network components.

No such queue size comparisons are required for the proportional fair optimization that is employed to define the Proportional Scheduler, and so the optimization can be completely decomposed as

$$
\max _{\sigma \in<\mathcal{S}>}\left\{\sum_{j \in \mathcal{J}} Q_{j} \log \sigma_{j}\right\}=\max _{\sigma \in<\mathcal{S}_{1}>}\left\{\sum_{j \in \mathcal{J}_{1}} Q_{j} \log \sigma_{j}\right\}+\max _{\sigma \in<\mathcal{S}_{2}>}\left\{\sum_{j \in \mathcal{J}_{2}} Q_{j} \log \sigma_{j}\right\}
$$

for complementary components having independent scheduling. This leads to more potential applications when the network decomposes, in comparison with the BackPressure policy.

\section{Maximum Stability Proof}

In this section, we prove Theorem 1, for which we employ fluid model techniques introduced in Dai (1995). The basic idea is to show that the Markov chain converges under appropriate scaling to a deterministic fluid model, and then to show that the queue lengths of all normalized fluid model solutions converge to 0 within a fixed time. This latter step requires most of the work in the proof and is shown by defining an appropriate Lyapunov function that decreases to 0 by this time.

From a mathematical point of view, the main contribution of this argument is the extension of fluid model techniques to a case with varying service speeds. Here, this requires identifying the Lyapunov function in 22 , showing it to be non-increasing in Corollary 2, and then showing that this Lyapunov function in fact decreases at a fixed rate by applying Lemmas 3 and 8 . The proofs of certain technical lemmas and propositions are relegated to the appendix. 


\subsection{Fluid Model}

We will employ, in Section 4, a fluid model, with the functions $A_{x}(t), D_{x}(t), Q_{x}(t)$, and $\Gamma_{k}(t)$, that satisfies the same conditions (3/8) as the discrete time switched network of Section 2, and such that $A_{x}(t), D_{x}(t)$ and $\Gamma_{k}(t)$ are non-negative and non-decreasing, and $Q_{x}(t)$ is non-negative, with $A_{x}(0)=D_{x}(0)=\Gamma_{k}(0)=0$ for $x \in \mathcal{J} \cup \mathcal{K} \cup \mathcal{R}$ and $k \in \mathcal{K}$. Note that each of the conditions (3) 8$)$ is invariant under the "law of large numbers" scaling, where both time and space are scaled equally.

In addition to these conditions, the route arrival and queue departure processes of the fluid model will be assumed to satisfy

$$
\begin{gathered}
A_{r}(t)=a_{r} t \\
Q_{j}(t)>0 \quad \text { implies } \quad D_{j}^{\prime}(t)=\sigma_{j}(\mathbf{Q}(t)),
\end{gathered}
$$

for given $a_{r}>0$ and for $t>s>0, j \in \mathcal{J}$ and $r \in \mathcal{R}$, with $\boldsymbol{\sigma}(\mathbf{Q})$ denoting a solution to the proportional fair optimization

$$
\boldsymbol{\sigma}(\mathbf{Q}) \in \underset{\boldsymbol{\sigma} \in<\mathcal{S}>}{\arg \max } \sum_{j \in \mathcal{J}} Q_{j} \log \sigma_{j}
$$

As in Section 2, we set $\sigma_{j}(\mathbf{Q})=0$ whenever $Q_{j}=0$ and, for each class $k \in r$ and queue $j \in \mathcal{J}$, set $a_{k}=a_{r}, a_{j}=\sum_{k \in j} a_{k}$, and $\boldsymbol{a}=\left(a_{j}: j \in \mathcal{J}\right)$. We will refer to the conditions (3-8) and (17, 19) on $\mathbf{A}(t), \mathbf{D}(t), \mathbf{Q}(t)$, and $\boldsymbol{\Gamma}(t)$ as proportional switch fluid model equations, or collectively, as the proportional switch fluid model (or fluid model, for short).

Together, the above conditions imply with a little work that, for $x \in \mathcal{J} \cup \mathcal{K} \cup \mathcal{R}$, the components $A_{x}(t), D_{x}(t), Q_{x}(t)$, and $\Gamma_{x}(t)$ are all Lipschitz continuous and thus almost everywhere differentiable. Note that $D_{j}^{\prime}(t)=0$ need not hold when $Q_{j}(t)=0$, since work may enter and leave an empty queue of a fluid model. Also note that a solution of the fluid model equations corresponding to a given initial condition need not be the unique such solution.

\subsection{Lyapunov Function and its Derivative}

In this subsection, we define the entropy function $H(t)$ and prove that it has negative derivative for non-empty subcritical systems. We first introduce the functions

$$
\begin{aligned}
L(t) & =\sum_{j \in \mathcal{J}} Q_{j}(t) \log D_{j}^{\prime}(t), \\
M(t) & =\sum_{j \in \mathcal{J}} \sum_{k \in j} \int_{D_{j}(t)}^{Q_{j}(0)+A_{j}(t)} \Gamma_{k}^{\prime}(s) \log \frac{\Gamma_{k}^{\prime}(s)}{a_{k}} d s ;
\end{aligned}
$$

$H(t)$ is then defined as

$$
H(t)=L(t)+M(t)
$$


The entropy function $H(t)$ builds on entropy functions in Massoulié (2007) and Bramson (1996). The term L(t) is derived in Massoulié (2007) as the large deviations rate function of a reversible network that approximates proportional fairness; the term $M(t)$ is similar to the entropy function employed in Bramson (1996) for FIFO multiclass queueing networks.

The function $L(t)$ is well defined everywhere (with, as earlier, the convention that $0 \log 0=0$ ). Note that

$$
\begin{aligned}
Q_{j}(t)=Q_{j}(0)+A_{j}(t)-D_{j}(t) & =\sum_{k \in j}\left(\Gamma_{k}\left(Q_{j}(0)+A_{j}(t)\right)-\Gamma_{k}\left(D_{j}(t)\right)\right) \\
& =\sum_{k \in j} \int_{D_{j}(t)}^{Q_{j}(0)+A_{j}(t)} \Gamma_{k}^{\prime}(s) d s
\end{aligned}
$$

the lower and upper bounds $D_{j}(t)$ and $Q_{j}(0)+A_{j}(t)$ for the integral in 23) can be thought of as the amounts of packet mass having departed from the queue $j$ by time $t$ and having departed by the later time when the last of the mass already at $j$ at time $t$ departs from the queue. With (23) in mind, note that $M(t)$ is the sum over the different queues of a weighted version of the packet mass at each queue $j$ at time $t$, based on the departure rates of the mass at the queues.

The following lemma shows that the function $H(t)$ is always non-negative for any solution of the fluid model.

Lemma 1. For any $t \geq 0, H(t) \geq 0$. Moreover, whenever the arrival rate vector a belongs to the interior of the scheduling set $\mathcal{C}$, then $H(t)=0$ iff $\mathbf{Q}(t)=0$.

Proof By 23,

$$
Q_{j}(t) \log a_{j}=\sum_{k \in j} \int_{D_{j}(t)}^{Q_{j}(0)+A_{j}(t)} \Gamma_{k}^{\prime}(s) \log a_{j} d s
$$

for each $j \in \mathcal{J}$; adding and subtracting such terms allows one to rewrite $H(t)$ as

$$
H(t)=\sum_{j \in \mathcal{J}} \int_{D_{j}(t)}^{Q_{j}(0)+A_{j}(t)} \sum_{k \in j} \Gamma_{k}^{\prime}(s) \log \left(\Gamma_{k}^{\prime}(s) \frac{a_{j}}{a_{k}}\right) d s+\sum_{j \in \mathcal{J}} Q_{j}(t) \log \frac{D_{j}^{\prime}(t)}{a_{j}} .
$$

By Jensen's inequality,

$$
\sum_{k \in j} \Gamma_{k}^{\prime}(s) \log \left(\Gamma_{k}^{\prime}(s) \frac{a_{j}}{a_{k}}\right)=-\sum_{k \in j} \Gamma_{k}^{\prime}(s) \log \left(\frac{1}{\Gamma_{k}^{\prime}(s)} \frac{a_{k}}{a_{j}}\right) \geq-\log \left(\sum_{k \in j} \Gamma_{k}^{\prime}(s) \frac{1}{\Gamma_{k}^{\prime}(s)} \frac{a_{k}}{a_{j}}\right)=0 .
$$

From this, it follows that the first summation in (24) is non-negative. The non-negativity of the second term in 24 follows since the service rates $D_{j}^{\prime}(t)$ solve the optimization problem (19) and $\boldsymbol{a} \in \mathcal{C}$, and so, in particular,

$$
\sum_{j \in \mathcal{J}} Q_{j}(t) \log D_{j}^{\prime}(t) \geq \sum_{j \in \mathcal{J}} Q_{j}(t) \log a_{j}
$$


Together with (25), this implies $H(t) \geq 0$.

When the fluid model is subcritical, one can improve on the inequality in the last display to show that $H(t)$ is positive when $\mathbf{Q}(t) \neq 0$. For this, note that $\boldsymbol{a} \in \mathcal{C}$ implies the existence of an $\epsilon>0$ such that

$$
(1+\epsilon) \boldsymbol{a} \in \mathcal{C}
$$

Since $\mathbf{D}^{\prime}(t)$ is an optimal scheduling, it follows that, for $\mathbf{Q}(t) \neq 0$,

$$
\sum_{j \in \mathcal{J}} Q_{j}(t) \log \frac{D_{j}^{\prime}(t)}{a_{j}} \geq \sum_{j \in \mathcal{J}} Q_{j}(t) \log (1+\epsilon)>0 .
$$

Consequently, $H(t)=0$ implies $Q(t)=0$. Trivially, $\mathbf{Q}(t)=0$ implies $H(t)=0$.

We next show that $H(t)$ is decreasing when $\mathbf{Q}(t) \neq 0$, by proving $H^{\prime}(t)$ is negative. Since we do not know in advance that $L(t)$ is sufficiently regular to apply the Fundamental Theorem of Calculus to $\int_{s}^{t} L^{\prime}(u) d u$, we will employ the following technical lemma, which is based on Lemma 5 of Massoulié (2007).

Lemma 2. There exists a time $T>0$ not depending on the initial queue state such that:

i) For $t \geq T|\mathbf{Q}(0)|$,

$$
L^{\prime}(t)=\sum_{j \in \mathcal{J}} Q_{j}^{\prime}(t) \log D_{j}^{\prime}(t) \quad \text { a.e. }
$$

ii) There exists a constant $\kappa_{L}>0$ such that, for all $t \geq s \geq 0$,

$$
L(t)-L(s) \leq \kappa_{L}(t-s)
$$

iii) For all $t \geq s \geq T|\mathbf{Q}(0)|$,

$$
L(t)-L(s) \leq \int_{s}^{t} L^{\prime}(u) d u
$$

A proof of this lemma is given in the Appendix.

Since the processes $\mathbf{A}(t)$ and $\mathbf{D}(t)$ are Lipschitz, it is easy to see that the term $M(t)$, which integrates an a.e. bounded function, is Lipschitz and hence a.e. differentiable. Thus, the following corollary is immediate from Lemma 2 .

Corollary 1. i) There exists a constant $\kappa_{H}>0$ such that, for $t \geq s \geq 0$,

$$
H(t)-H(s) \leq \kappa_{H}(t-s)
$$

ii) There exists a time $T>0$ not depending on the initial state such that $H(t)$ is a.e. differentiable on $t \in[T|\mathbf{Q}(0)|, \infty)$ and, for given $s, t$ with $t \geq s \geq T|\mathbf{Q}(0)|$,

$$
H(t)-H(s) \leq \int_{s}^{t} H^{\prime}(u) d u
$$


We now focus on computing $H^{\prime}(t)$. The following proposition shows that $H^{\prime}(t)$ is the sum of two terms: the (unnormalized) relative entropy between route departure and arrival rates, and the (unnormalized) relative entropy between queue arrival and departure rates. This decomposition will play an important role in the proof of Theorem 2

Proposition 1. For $T$ as in Corollary 1 , and $t \geq T|\mathbf{Q}(0)|$,

$$
H^{\prime}(t)=-\sum_{r \in \mathcal{R}} D_{r}^{\prime}(t) \log \frac{D_{r}^{\prime}(t)}{A_{r}^{\prime}(t)}-\sum_{j \in \mathcal{J}} A_{j}^{\prime}(t) \log \frac{A_{j}^{\prime}(t)}{D_{j}^{\prime}(t)} \quad \text { a.e. }
$$

As earlier, we set $0 \log 0=0$; we also set $0 \log (0 / 0)=0$ here and note that the set where $\left\{A_{j}^{\prime}(t)>\right.$ $\left.0, D_{j}^{\prime}(t)=0\right\}$ has measure 0 for each $j$. For brevity, when no confusion is likely, we will often omit the quantifier "a.e." in our computations.

Proof of Proposition 1 Differentiating the expression for $H(t)$ in 22 and applying (27) gives

$$
\begin{aligned}
H^{\prime}(t)= & \sum_{j \in \mathcal{J}} \sum_{k \in j} A_{j}^{\prime}(t) \Gamma_{k}^{\prime}\left(A_{j}(t)\right) \log \frac{\Gamma_{k}^{\prime}\left(A_{j}(t)\right)}{a_{k}} \\
& -\sum_{j \in \mathcal{J}} \sum_{k \in j} D_{j}^{\prime}(t) \Gamma_{k}^{\prime}\left(D_{j}(t)\right) \log \frac{\Gamma_{k}^{\prime}\left(D_{j}(t)\right)}{a_{k}}+\sum_{j \in \mathcal{J}} Q_{j}^{\prime}(t) \log D_{j}^{\prime}(t) .
\end{aligned}
$$

On the other hand, differentiation of the expressions (6) and (7) implies that

$$
D_{k}^{\prime}(t)=D_{j}^{\prime}(t) \Gamma_{k}^{\prime}\left(D_{j}(t)\right), \quad A_{k}^{\prime}(t)=A_{j}^{\prime}(t) \Gamma_{k}^{\prime}\left(A_{j}(t)\right),
$$

and substitution of these terms into $H^{\prime}(t)$ gives

$$
\begin{aligned}
H^{\prime}(t)= & \sum_{j \in \mathcal{J}} \sum_{k \in j} A_{k}^{\prime}(t) \log \frac{A_{k}^{\prime}(t)}{A_{j}^{\prime}(t) a_{k}}-\sum_{j \in \mathcal{J}} \sum_{k \in j} D_{k}^{\prime}(t) \log \frac{D_{k}^{\prime}(t)}{D_{j}^{\prime}(t) a_{k}} \\
& +\sum_{j \in \mathcal{J}}\left(A_{j}^{\prime}(t)-D_{j}^{\prime}(t)\right) \log D_{j}^{\prime}(t) \\
= & \sum_{j \in \mathcal{J}} \sum_{k \in j}\left(A_{k}^{\prime}(t) \log \frac{A_{k}^{\prime}(t)}{a_{k}}-D_{k}^{\prime}(t) \log \frac{D_{k}^{\prime}(t)}{a_{k}}\right)+\sum_{j \in \mathcal{J}} A_{j}^{\prime}(t) \log \frac{D_{j}^{\prime}(t)}{A_{j}^{\prime}(t)} .
\end{aligned}
$$

Application of (8) shows that the double sum above telescopes over the classes in each route; since $A_{r}^{\prime}(t) \equiv a_{k}$ for each $k \in r$, only the terms due to external departures $D_{r}^{\prime}(t)$ do not cancel out, which implies (31).

It is now easy to see that $H^{\prime}(t) \leq 0$ for suitably large $t$.

Corollary 2. For $T$ as in Corollary 1 and $t>T|\mathbf{Q}(0)|, H^{\prime}(t) \leq 0$ a.e.

Proof For each choice of $a, d \geq 0$, one has $a \log (a / d) \geq a-d$ (as before, $0 \log 0=0,0 \log (0 / 0)=0)$. Applying this to 31 implies that

$$
H^{\prime}(t) \leq \sum_{r \in \mathcal{R}}\left(A_{r}^{\prime}(t)-D_{r}^{\prime}(t)\right)-\sum_{j \in \mathcal{J}}\left(A_{j}^{\prime}(t)-D_{j}^{\prime}(t)\right)=\sum_{r \in \mathcal{R}} Q_{r}^{\prime}(t)-\sum_{j \in \mathcal{J}} Q_{j}^{\prime}(t)=0
$$

with the final equality following since the rates of change in total queue size summed over all queues and summed over all routes are equal. 


\subsection{Fluid Stability and Positive Recurrence}

We wish to show that, in an appropriate averaged sense, $H(t)$ is always decreasing at a rate bounded away from zero when $\mathbf{Q}(t) \neq 0$. It will follow quickly from this that $\mathbf{Q}(t)=0$ for $t \geq \gamma|\mathbf{Q}(0)|$, with $\gamma$ not depending on the particular fluid model solution; when this behavior occurs, the fluid model is said to be stable.

The following lemma shows that, for subcritical networks, queue sizes must vary over time and hence there is a "mismatch" between arrival rates and departure rates at some of the queues. As we will see, this together with the relative entropy terms in Proposition 1 will show the Lyapunov function $H(t)$ decreases to 0 at a uniform rate. The lemma is proven in Subsection B of the Appendix.

Lemma 3. Assume that the arrival rate vector $\boldsymbol{a} \in \mathcal{C}$. Then there exist constants $c, \kappa>0$ such that, whenever $|\mathbf{Q}(0)|>0$,

$$
\left|Q_{j}(t)-Q_{j}(0)\right| \geq \kappa|\mathbf{Q}(0)|
$$

for some $j \in \mathcal{J}$ and $t \leq c|\mathbf{Q}(0)|$.

The following elementary lemma is also proved in the Appendix.

Lemma 4. For $x, y \in(0, K]$ and any $K>0$,

$$
y \log \left(\frac{y}{x}\right)-(y-x) \geq \frac{1}{2 K}(y-x)^{2} .
$$

We will use the two preceding lemmas to give the following lower bound on the rate of decrease of $H(t)$. The basic argument for the proposition is similar to that leading up to (4.24) in Bramson (1996), although the reasoning differs in a few ways.

Proposition 2. Assume that the arrival rate vector $\boldsymbol{a}$ belongs to the subcritical region $\mathcal{C}$. For $T$ as in Corollary 1 , there exist constants $c_{1}, c_{2}>0$ such that, for $t \geq T|\mathbf{Q}(0)|$,

$$
H\left(t+c_{1}|\mathbf{Q}(t)|\right)-H(t) \leq-c_{2}|\mathbf{Q}(t)|
$$

Proof Let $K$ be the Lipschitz constant bounding the processes $D_{r}^{\prime}(t), A_{r}^{\prime}(t), D_{j}^{\prime}(t)$, and $A_{j}^{\prime}(t)$, over all $r \in \mathcal{R}$ and $j \in \mathcal{J}$. From Proposition 1 and Lemma 4 .

$$
\begin{aligned}
H^{\prime}(t) \leq & -\sum_{r \in \mathcal{R}} \frac{1}{2 K}\left(D_{r}^{\prime}(t)-A_{r}^{\prime}(t)\right)^{2}-\sum_{j \in \mathcal{J}} \frac{1}{2 K}\left(A_{j}^{\prime}(t)-D_{j}^{\prime}(t)\right)^{2} \\
& -\sum_{r \in \mathcal{R}}\left(D_{r}^{\prime}(t)-A_{r}^{\prime}(t)\right)-\sum_{j \in \mathcal{J}} Q_{j}^{\prime}(t) \\
= & -\sum_{r \in \mathcal{R}} \frac{1}{2 K}\left(D_{r}^{\prime}(t)-A_{r}^{\prime}(t)\right)^{2}-\sum_{j \in \mathcal{J}} \frac{1}{2 K}\left(Q_{j}^{\prime}(t)\right)^{2} \leq-\frac{1}{2 K} \sum_{j \in \mathcal{J}}\left(Q_{j}^{\prime}(t)\right)^{2} .
\end{aligned}
$$


On account of (30) in Corollary 1, this implies

$$
H(t)-H(s) \leq-\frac{1}{2 K} \sum_{j \in \mathcal{J}} \int_{s}^{t}\left(Q_{j}^{\prime}(u)\right)^{2} d u .
$$

By Lemma 3, there exists a $j \in \mathcal{J}$ and a value of $t$ with $t \leq s+c_{1}|\mathbf{Q}(s)|$ such that

$$
\left|Q_{j}(t)-Q_{j}(s)\right| \geq \kappa|\mathbf{Q}(s)|
$$

It therefore follows from the Cauchy-Schwarz Inequality that

$$
\kappa|\mathbf{Q}(s)| \leq \int_{s}^{s+c_{1}|\mathbf{Q}(s)|}\left|Q_{j}^{\prime}(u)\right| d u \leq\left(\int_{s}^{s+c_{1}|\mathbf{Q}(s)|}\left(Q_{j}^{\prime}(u)\right)^{2} d u\right)^{\frac{1}{2}} \sqrt{c_{1}|\mathbf{Q}(s)|}
$$

for this choice of $j$, and hence

$$
\int_{s}^{s+c_{1}|\mathbf{Q}(s)|}\left(Q_{j}^{\prime}(u)\right)^{2} d u \geq \frac{\kappa^{2}}{c_{1}}|\mathbf{Q}(s)| .
$$

Applying the last inequality to (35), one obtains

$$
H\left(s+c_{1}|\mathbf{Q}(s)|\right)-H(s) \leq-\frac{\kappa^{2}}{2 K c_{1}}|\mathbf{Q}(s)|,
$$

and so (34) follows by setting $c_{2}=\kappa^{2} / 2 K c_{1}$.

Employing Lemma 1, Corollary 1, and Proposition 2, we now show our main result for proportional switch fluid models.

THEOREM 2. If $\boldsymbol{a} \in \mathcal{C}$, then the proportional switch fluid model is stable.

Proof The proof follows the reasoning in Bramson (1996), which we repeat for completeness. Let $c_{1}$ and $c_{2}$ be as in in Proposition 2, set $t_{0}=T|\mathbf{Q}(0)|$ for $T$ as in Corollary 1 , and define

$$
t_{i+1}=t_{i}+c_{1}\left|\mathbf{Q}\left(t_{i}\right)\right| .
$$

By Proposition 2, since $H(t) \geq 0$,

$$
H\left(t_{0}\right) \geq H\left(t_{0}\right)-H\left(t_{i+1}\right) \geq \sum_{l=0}^{i}\left(H\left(t_{l}\right)-H\left(t_{l+1}\right)\right) \geq \sum_{l=0}^{i} c_{2}\left|\mathbf{Q}\left(t_{l}\right)\right|=\sum_{l=0}^{i} \frac{c_{2}}{c_{1}}\left(t_{l+1}-t_{l}\right)=\frac{c_{2}}{c_{1}}\left(t_{i+1}-t_{0}\right),
$$

and so, as $i \rightarrow \infty$, one has $t_{\infty}=\lim _{i \rightarrow \infty} t_{i} \leq c_{1} H\left(t_{0}\right) / c_{2}+t_{0}<\infty$. By the continuity of $\mathbf{Q}(t)$ and the definition of $t_{\infty}, \mathbf{Q}\left(t_{\infty}\right)=0$, and consequently, since $H(t)$ is non-increasing,

$$
H(t)=0 \quad \text { for } t \geq \frac{c_{1}}{c_{2}} H\left(t_{0}\right)+t_{0} .
$$

On the other hand, by Corollary 1, $H\left(t_{0}\right) \leq \kappa_{H} t_{0}=\kappa_{H} T|\mathbf{Q}(0)|$. Setting $c_{3}=c_{1} \kappa_{H} T / c_{2}+T$, this together with Lemma 1 implies

$$
\mathbf{Q}(t)=0 \quad \text { for } t \geq c_{3}|\mathbf{Q}(0)|
$$

as required. 
The main result in the paper, Theorem 1, follows immediately from Theorem 2 and the following proposition.

Proposition 3. Suppose that the proportional switch fluid model is stable. Then the corresponding proportional switched network is positive recurrent.

We postpone the proof of Proposition 3 to Subsection $\mathrm{D}$ of the Appendix. We will follow there the approach of Dai (1995) as presented in Bramson (2008), although the argument is considerably shorter in the present setting and requires only several pages.

\section{References}

Andrews, M., Kumaran, K., Ramanan, K., Stolyar, A., Vijayakumar, R., and Whiting, P. (2004). Scheduling in a queuing system with asynchronously varying service rates. Probability in the Engineering and Informational Sciences, 18(2):191-217.

Andrews, M. and Zhang, L. (2003). Achieving stability in networks of input-queued switches. Networking, IEEE/ACM Transactions on, 11(5):848-857.

Anselmi, J., D'Auria, B., and Walton, N. (2013). Closed queueing networks under congestion: Nonbottleneck independence and bottleneck convergence. Mathematics of Operations Research, 38(3):469-491.

Appenzeller, G., Keslassy, I., and McKeown, N. (2004). Sizing router buffers. In Proceedings of SIGCOMM '04, pages 281-292, New York, NY, USA. ACM.

Armony, M. and Bambos, N. (2003). Queueing dynamics and maximal throughput scheduling in switched processing systems. Queueing systems, 44(3):209-252.

Baskett, F., Chandy, K., Muntz, R., and Palacios, F. (1975). Open, closed, and mixed networks of queues with different classes of customers. J. ACM, 22(2):248-260.

Billingsley, P. (1999). Convergence of Probability Measures. Wiley, New York.

Bonald, T. and Massoulié, L. (2001). Impact of fairness on internet performance. Proc. of ACM Sigmetrics, 29:82-91.

Bonald, T. and Proutière, A. (2002). Insensitivity in processor-sharing networks. Performance Evaluation, 49:193-203.

Bonald, T. and Proutière, A. (2003). Insensitive bandwidth sharing in data networks. Queueing Systems, 44:69-100.

Bonald, T. and Proutière, A. (2004). On performance bounds for balanced fairness. Performance Evaluation, $55: 25-50$.

Borodin, A., Kleinberg, J., Raghavan, P., Sudan, M., and Williamson, D. (2001). Adversarial queuing theory. J. ACM, 48:13-38.

Bramson, M. (1994). Instability of FIFO queueing networks. The Annals of Applied Probability, 4(2):414-431. 
Bramson, D'Auria and Walton: Proportional Switching

Article submitted to Operations Research; manuscript no. (Please, provide the manuscript number!)

Bramson, M. (1996). Convergence to equilibria for fluid models of FIFO queueing networks. Queueing Systems Theory Appl., 22(1-2):5-45.

Bramson, M. (2008). Stability of queueing networks. Probab. Surv., 5:169-345.

Bui, L., Srikant, R., and Stolyar, A. (2011). A novel architecture for reduction of delay and queueing structure complexity in the back-pressure algorithm. IEEE/ACM Trans. Netw., 19(6):1597-1609.

Burke, P. (1956). The output of a queuing system. Operations Research, 4(6):699-704.

Dai, J. (1995). On positive harris recurrence of multiclass queueing networks: a unified approach via fluid limit models. The Annals of Applied Probability, 5(1):49-77.

Dai, J. and Lin, W. (2005). Maximum pressure policies in stochastic processing networks. Operations Research, 53(2):197-218.

De Veciana, G., Lee, T., and Konstantopoulos, T. (1999). Stability and performance analysis of networks supporting services with rate control - could the internet be unstable? IEEE/ACM Trans. Networking, $9(1): 2-14$.

Dieker, A. and Shin, J. (2013). From local to global stability in stochastic processing networks through quadratic lyapunov functions. Mathematics of Operations Research, 38(4):638-664.

Eryilmaz, A., Srikant, R., and Perkins, J. (2005). Stable scheduling policies for fading wireless channels. Networking, IEEE/ACM Transactions on, 13(2):411-424.

Georgiadis, L., Neely, M., and Tassiulas, L. (2006). Resource allocation and cross-layer control in wireless networks. Now Publishers Inc.

Gromoll, H. and Williams, R. (2009). Fluid limits for networks with bandwidth sharing and general document size distributions. Annals of Applied Probability, 19:243-280.

Harrison, J., Mandayam, C., Shah, D., and Yang, Y. (2014). Resource sharing nertworks: overview and an open problem. Stochastic Systems, 4(2):524-555.

Hsu, J. and Burke, P. (1976). Behavior of tandem buffers with geometric input and markovian output. Communications, IEEE Transactions on, 24(3):358-361.

Jackson, J. (1963). Jobshop-like queueing systems. Management science, 10(1):131-142.

Jagannathan, K., Markakis, M., Modiano, E., and Tsitsiklis, J. (2011). Queue length asymptotics for generalized max-weight scheduling in the presence of heavy-tailed traffic. In INFOCOM, 2011 Proceedings IEEE, pages $2318-2326$.

Ji, B., Joo, C., and Shroff, N. (2013). Throughput-optimal scheduling in multihop wireless networks without per-flow information. Networking, IEEE/ACM Transactions on, 21(2):634-647.

Jonckheere, M. and López, S. (2014). Large deviations for the stationary measure of networks under proportional fair allocations. Mathematics of Operations Research, 39(2):418-431. 
Kang, W., Kelly, F., Lee, N., and Williams, R. (2007). Product form stationary distributions for diffusion approximations to a flow level model operating under a proportional fair sharing policy. Performance Evaluation Review, 35:36-38.

Kang, W., Kelly, F., Lee, N., and Williams, R. J. (2009). State space collapse and diffusion approximation for a network operating under a fair bandwidth sharing policy. Ann. Appl. Probab., 19:1719-1780.

Kannan, R. and Krueger, C. (1996). Advanced analysis: on the real line. Universitext Series. Springer.

Kelly, F. (1975). Networks of queues with customers of different types. Journal of Applied Probability, 12(3):542-554.

Kelly, F. (1979). Reversibility and Stochastic Networks. Wiley, Chicester.

Kelly, F. (1997). Charging and rate control for elastic traffic. European transactions on Telecommunications, 8(1):33-37.

Kelly, F., Massoulié, L., and Walton, N. (2009). Resource pooling in congested networks: proportional fairness and product form. Queueing Systems, 63(1-4):165-194.

Kelly, F. and Williams, R. (2004). Fluid model for a network operating under a fair bandwidth-sharing policy. The Annals of Applied Probability, 14(3):1055-1083.

Kelly, F. P. (1989). On a class of approximations for closed queueing networks. Queueing Systems, 4(1):69-76.

Kushner, H. and Whiting, P. (2004). Convergence of proportional-fair sharing algorithms under general conditions. Wireless Communications, IEEE Transactions on, 3(4):1250-1259.

Li, B. and Srikant, R. (2016). Queue-proportional rate allocation with per-link information in multihop wireless networks. Queueing systems (to appear).

Lu, S. H. and Kumar, P. (1991). Distributed scheduling based on due dates and buffer priorities. Automatic Control, IEEE Transactions on, 36(12):1406-1416.

Mandelbaum, A. and Stolyar, A. (2004). Scheduling flexible servers with convex delay costs: Heavy-traffic optimality of the generalized c $\mu$-rule. Operations Research, 52(6):836-855.

Markakis, M. G., Modiano, E., and Tsitsiklis, J. N. (2014). Max-weight scheduling in queueing networks with heavy-tailed traffic. IEEE/ACM Transactions on Networking (TON), 22(1):257-270.

Massoulié, L. (2007). Structural properties of proportional fairness: Stability and insensitivity. The Annals of Applied Probability, 17(3):809-839.

Massoulié, L. and Roberts, J. (1999). Bandwidth sharing: Objectives and algorithms. IEEE Infocom 1999, 10(3):320-328.

Massoulie, L. and Roberts, J. W. (2000). Bandwidth sharing and admission control for elastic traffic. Telecommunication systems, 15(1-2):185-201.

McKeown, N. (1999). The islip scheduling algorithm for input-queued switches. Networking, IEEE/ACM Transactions on, 7(2):188-201. 
Bramson, D'Auria and Walton: Proportional Switching

McKeown, N., Mekkittikul, A., Anantharam, V., and Walrand, J. (1999). Achieving 100\% throughput in an input-queued switch. Communications, IEEE Transactions on, 47(8):1260-1267.

Meyn, S. (2009). Stability and asymptotic optimality of generalized maxweight policies. SIAM Journal on Control and Optimization, 47(6):3259-3294.

Mo, J. and Walrand, J. (2000). Fair end-to-end window-based congestion control. IEEE/ACM Transactions on Networking (ToN), 8(5):556-567.

Paganini, F., Tang, A., Ferragut, A., and Andrew, L. (2012). Network stability under alpha fair bandwidth allocation with general file size distribution. Automatic Control, IEEE Transactions on, 57(3):579-591.

Rybko, A. and Stolyar, A. (1992). Ergodicity of stochastic processes describing the operation of open queueing networks. Problemy Peredachi Informatsii, 28(3):3-26.

Schweitzer, P. (1979). Approximate analysis of multiclass closed networks of queues. In Proceedings of the international conference on stochastic control and optimization, Free University, Amsterdam.

Shah, D. and Wischik, D. (2011). Fluid models of congestion collapse in overloaded switched networks. Queueing Syst., 69(2):121-143.

Shah, D. and Wischik, D. (2012). Switched networks with maximum weight policies: Fluid approximation and multiplicative state space collapse. The Annals of Applied Probability, 22(1):70-127.

Stolyar, A. (2004). Maxweight scheduling in a generalized switch: State space collapse and workload minimization in heavy traffic. The Annals of Applied Probability, 14(1):1-53.

Stolyar, A. (2011). Large number of queues in tandem: Scaling properties under back-pressure algorithm. Queueing Syst. Theory Appl., 67(2):111-126.

Tassiulas, L. and Ephremides, A. (1992). Stability properties of constrained queueing systems and scheduling policies for maximum throughput in multihop radio networks. Automatic Control, IEEE Transactions on, 37(12):1936-1948.

Varaiya, P. (2013). Max pressure control of a network of signalized intersections. Transportation Research Part C: Emerging Technologies, 36(0):177-195.

Venkataramanan, V. and Lin, X. (2007). Structural properties of ldp for queue-length based wireless scheduling algorithms. In Proceedings of Allerton.

Viswanath, P., Tse, D., and Laroia, R. (2002). Opportunistic beamforming using dumb antennas. Information Theory, IEEE Transactions on, 48(6):1277-1294.

Vlasiou, M., Zhang, J., and Zwart, B. (2014). Insensitivity of proportional fairness in critically loaded bandwidth sharing networks. (preprint).

Walton, N. (2011). Insensitive, maximum stable allocations converge to proportional fairness. Queueing Systems, 68:51-60. 
Walton, N. S. (2009). Proportional fairness and its relationship with multi-class queueing networks. Ann. Appl. Probab., 22(6):2301-2333.

Whittle, P. (1985). Partial balance and insensitivity. Journal of Applied Probability, 22(1):168-176.

Williams, D. (1991). Probability with Martingales. Cambridge University Press.

Ye, H.-Q., Ou, J., and Yuan, X.-M. (2005). Stability of data networks: Stationary and bursty models. Operations Research, 53(1):107-125.

Ye, H.-Q. and Yao, D. (2012). A stochastic network under proportional fair resource control-diffusion limit with multiple bottlenecks. Operations Research, 60(3):716-738.

Ying, L., Srikant, R., Towsley, D., and Liu, S. (2011). Cluster-based back-pressure routing algorithm. Networking, IEEE/ACM Transactions on, 19(6):1773-1786.

Zachary, S. (2007). A note on insensitivity in stochastic networks. J. Appl. Probab., 44(1):238-248.

Zhong, Y. (2012). Resource Allocation in Stochastic Processing Networks: Performance and Scaling. PhD thesis, MIT.

\section{Appendix A: Lemmas from Section 4.2}

The main aim of this section is to prove Lemma 2. We first present the supporting Lemmas 5 - 7 . Lemma 5 is proved in Lemma A.3 of Kelly and Williams (2004).

Lemma 5. The function $\mathbf{Q} \mapsto \sigma_{j}(\mathbf{Q})$ is continuous on the set $\left\{\mathbf{Q}: Q_{j}>0\right\}$.

When $Q_{j} /|\mathbf{Q}|$ is bounded away from 0, Lemma 6 states that the corresponding coordinate $\sigma_{j}(\mathbf{Q})$ of the proportional fair optimization in $(19)$ is also bounded away from 0 .

Lemma 6. Let $\boldsymbol{\sigma}(\mathbf{Q})$ be a solution to the proportional fair optimization $(19)$, where $|\mathbf{Q}|>0$. Then, for any $\epsilon>0$, there exists a $\delta>0$ such that, for any $j, \sigma_{j}(\mathbf{Q}) \geq \delta$ whenever $Q_{j} \geq \epsilon|\mathbf{Q}|$.

Proof Scaling $\mathbf{Q}$ by $|\mathbf{Q}|$, it suffices to prove the result for $\sum_{j^{\prime} \in \mathcal{J}} Q_{j^{\prime}}=1$, when $Q_{j} \geq \epsilon$. If the result were not true, then, for some $\epsilon>0$ and $j$, there would exist a sequence $\mathbf{Q}^{(k)}$ with $Q_{j}^{(k)}>\epsilon$ and $\sigma_{j}\left(\mathbf{Q}^{(k)}\right) \rightarrow 0$ as $k \rightarrow \infty$. It would follow that

$$
\sum_{j^{\prime} \in \mathcal{J}} Q_{j^{\prime}}^{(k)} \log \sigma_{j^{\prime}}\left(\mathbf{Q}^{(k)}\right) \leq Q_{j}^{(k)} \log \sigma_{j}\left(\mathbf{Q}^{(k)}\right)+\left(1-Q_{j}^{(k)}\right) \log \sigma_{\max } \underset{k \rightarrow \infty}{\longrightarrow}-\infty
$$

where $\sigma_{\max }$ is defined at the beginning of Subsection 2.1. Since the maximum in $(19)$ is bounded from below (by any fixed choice of $\boldsymbol{\sigma}$ ), this gives a contradiction.

Lemma 7 bounds $D_{k}(t) / t$ from below for large $t$. A related result is given in Lemma 5 of Massoulié (2007).

Lemma 7. There exists $T>0$, not depending on $\mathbf{Q}(0)$, such that, for any class $k$ and $t \geq T|\mathbf{Q}(0)|$, i) for appropriate $\beta>0, D_{k}(t)>\beta t$ and ii) $D_{k}^{\prime}(t)>0$ a.e. 
Proof i) We argue by induction along each route, assuming for a class $k$, times $s$ with $s \geq$ $T_{1}|\mathbf{Q}(0)|$ for a given $T_{1} \geq 1$, and a given $\alpha>0$, that $A_{k}(s) \geq \alpha s$. We first show the analog of the desired result, but for $D_{j}(t)$ instead of $D_{k}(t)$, where $k \in j$. If $D_{j}(s)<\alpha s / 2$, then $Q_{j}(s) \geq Q_{k}(s) \geq$ $\alpha s / 2$, and so, when $s \geq T_{1}|\mathbf{Q}(0)|$,

$$
\frac{Q_{j}(s)}{\sum_{j^{\prime} \in \mathcal{J}} Q_{j^{\prime}}} \geq \frac{\alpha s / 2}{\sum_{j^{\prime} \in \mathcal{J}}\left(a_{j^{\prime}} s+Q_{j^{\prime}}(0)\right)} \geq \epsilon
$$

for appropriate $\epsilon>0$. Therefore, by Lemma $6, D_{j}^{\prime}(s) \geq \delta$, where $\delta$ does not depend on the initial queue size distribution; from this, it follows that, if $D_{j}(s)<\alpha s / 2$ for all $s \in[t / 2, t]$ with $t \geq$ $2 T_{1}|\mathbf{Q}(0)|$, then $D_{j}(t) \geq \delta t / 2$. Combining the last inequality with the case when $D_{j}(s) \geq \alpha s / 2$ for some $s \in[t / 2, t]$, and setting $\delta^{\prime}=\min \{\alpha / 4, \delta / 2\}$, one obtains $D_{j}(t) \geq \delta^{\prime} t$ for $t \geq 2 T_{1}|\mathbf{Q}(0)|$ in both cases.

We now show $D_{k}(t)$ also increases linearly in time. Note that $A_{j}(t) \leq \bar{a} t$, where $\bar{a}=\max _{j} a_{j}+$ $|\mathcal{K}|\left|\sigma_{\max }\right|$. Applying this and setting $\gamma=\delta^{\prime} / 2 \bar{a}$, one can check that $D_{j}(t) \geq A_{j}(\gamma t)+|\mathbf{Q}(0)|$ for $t \geq T_{2}|\mathbf{Q}(0)|$, with $T_{2}=2 \max \left(T_{1}, 1 / \delta^{\prime}\right)$. Using (6) and the FIFO assumption (7), one therefore obtains

$$
D_{k}(t)=\Gamma_{k}\left(D_{j}(t)\right) \geq \Gamma_{k}\left(A_{j}(\gamma t)+Q_{j}(0)\right)=A_{k}(\gamma t)+Q_{k}(0) \geq \gamma \alpha t
$$

for $t \geq T_{2}|\mathbf{Q}(0)|$, where the last inequality follows from the induction assumption. Since $A_{k}(t)=a_{r} t$ for the first class on a route $r$, applying (8) and the argument just given, we can inductively show that for all classes on a given route $r, D_{k}(t)>\beta t$ for $t \geq T|\mathbf{Q}(0)|$, for appropriate choices of $\beta, T>0$. This is the desired result.

ii) We again argue by induction along each route, assuming for a class $k$ that $A_{k}^{\prime}(t)>0$ a.e. on $t \geq T_{3}|\mathbf{Q}(0)|$ for some $T_{3} \geq T$, where $T$ is given in part i); we henceforth restrict our attention to times $t$ where the arrival and departure processes are differentiable. It follows under this assumption that

$$
D_{j}^{\prime}(t)>0 \quad \text { for } t \geq T_{3}|\mathbf{Q}(0)|
$$

because, if $Q_{j}(t)>0$, then $D_{j}^{\prime}(t)>0$ by Lemma 6, whereas, if $Q_{j}(t)=0$, then $Q_{j}^{\prime}(t)=0$ and so $D_{j}^{\prime}(t)=A_{j}^{\prime}(t) \geq A_{k}^{\prime}(t)>0$.

By (7) and the definition of $\Gamma_{k}^{\prime}$, for $t \geq T_{3}|\mathbf{Q}(0)|$,

$$
\Gamma_{k}^{\prime}\left(A_{j}(t)+Q_{j}(0)\right)=\frac{A_{k}^{\prime}(t)}{A_{j}^{\prime}(t)}>0 .
$$

Also, by part i), for $t \geq T_{4}|\mathbf{Q}(0)|$, with $T_{4}=\max \left(T, \beta^{-1}\left(A_{j}\left(T_{3}|\mathbf{Q}(0)|\right) /|\mathbf{Q}(0)|+1\right)\right)$, one has $D_{j}(t) \geq$ $A_{j}\left(T_{3}|\mathbf{Q}(0)|\right)+Q_{j}(0)$; together with $(39)$, this implies that

$$
\Gamma_{k}^{\prime}\left(D_{j}(t)\right)>0
$$


for $t \geq T_{4}|\mathbf{Q}(0)|$. Using the same upper bound for $A_{j}(t)$ as in part i), one can show that $T_{4} \leq T_{5}$ for $T_{5}=\max \left(T_{3}, \beta^{-1}\left(\bar{a} T_{3}+1\right)\right)$. So, 38$)$ and 40 together imply that

$$
D_{k}^{\prime}(t)=D_{j}^{\prime}(t) \Gamma_{k}^{\prime}\left(D_{j}(t)\right)>0
$$

for $t \geq T_{5}|\mathbf{Q}(0)|$. Applying (8), one can then inductively repeat this argument along the classes of each route to give the desired result after a new choice of $T$.

We now employ Lemmas 5 and 7 to demonstrate Lemma 2 .

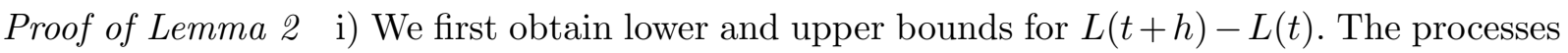
$\mathbf{D}(t)$ and $\mathbf{Q}(t)$ are almost everywhere differentiable and, by part ii) of Lemma 7 , there exists a $T>0$ such that $D_{j}^{\prime}(t)>0$ a.e. on $t \geq T|\mathbf{Q}(0)|$ for each queue $j$; choose a $t$ satisfying these conditions. Since $\mathbf{D}^{\prime}(t)$ is suboptimal for the proportional fair optimization $(19)$ with queue size vector $\mathbf{Q}(t+h)$ for given $h$,

$$
\begin{aligned}
L(t+h)-L(t) & =\sum_{j \in \mathcal{J}}\left(Q_{j}(t+h) \log D_{j}^{\prime}(t+h)-Q_{j}(t) \log D_{j}^{\prime}(t)\right) \\
& \geq \sum_{j \in \mathcal{J}}\left(Q_{j}(t+h)-Q_{j}(t)\right) \log D_{j}^{\prime}(t) .
\end{aligned}
$$

Also, since $\mathbf{D}^{\prime}(t+h)$ is suboptimal for queue size vector $\mathbf{Q}(t)$,

$$
\begin{aligned}
L(t+h)-L(t) & \leq \sum_{j \in \mathcal{J}}\left(Q_{j}(t+h)-Q_{j}(t)\right) \log D_{j}^{\prime}(t+h) \\
& \leq \sum_{\substack{j \in \mathcal{J}: \\
Q_{j}(t)>0}}\left(Q_{j}(t+h)-Q_{j}(t)\right) \log D_{j}^{\prime}(t+h)+\sum_{\substack{j \in \mathcal{J}: \\
Q_{j}(t)=0}} Q_{j}(t+h) \log \sigma_{\max } .
\end{aligned}
$$

(After splitting the sum over $j$ into two parts depending on whether or not $Q_{j}(t)=0$, we employed $\log D_{j}^{\prime}(t) \leq \log \sigma_{\max }$ in the summation over $j$ with $Q_{j}(t)=0$.)

After dividing by $h$, we consider the limiting behavior of the left hand sides of (41) and (42), as $h \rightarrow 0$, by employing the bounds on the right hand sides of the equations. Since $\mathbf{Q}^{\prime}(t)$ exists, after dividing by $h$, the right hand side of (41) converges to

$$
\sum_{j \in \mathcal{J}} Q_{j}^{\prime}(t) \log D_{j}^{\prime}(t)
$$

as $h \rightarrow 0$. On the other hand, by Lemma 5 , $D_{j}^{\prime}(t)=\sigma_{j}(\mathbf{Q}(t))$ is continuous where $Q_{j}(t)>0$, and so $D_{j}^{\prime}(t+h) \rightarrow D_{j}^{\prime}(t)$ as $h \rightarrow 0$. Recalling that $Q_{j}^{\prime}(t)=0$ when $Q_{j}(t)=0$, the first term on the right hand side of (42) also converges to 43) after dividing by $h$. Moreover, the last term in (42) converges to 0 after dividing by $h$, since $Q_{j}^{\prime}(t)=0$ for such $j$. Putting these limits together, (27) follows. 
ii) Most of the work consists of computing an upper bound for the upper right Dini derivative of the function $L(\cdot)$ at time $t$, which is given by

$$
D^{+} L(t)=\limsup _{h \searrow 0} \frac{L(t+h)-L(t)}{h} .
$$

By the same reasoning as in 42 , for $h>0$,

$$
\begin{aligned}
\frac{L(t+h)-L(t)}{h} & \leq \sum_{j \in \mathcal{J}} \frac{Q_{j}(t+h)-Q_{j}(t)}{h} \log D_{j}^{\prime}(t+h) \\
& \leq \sum_{\substack{j \in \mathcal{J}: \\
Q_{j}(t)=0}} \frac{Q_{j}(t+h)}{h} \log D_{j}^{\prime}(t+h)+\sum_{\substack{j \in \mathcal{J}: \\
Q_{j}(t)>0}} \frac{Q_{j}(t+h)-Q_{j}(t)}{h} \log D_{j}^{\prime}(t+h) .
\end{aligned}
$$

Denoting by $K_{A}$ and $K_{Q}$ the Lipschitz constants for the processes $\mathbf{A}(t)$ and $\mathbf{Q}(t)$, and employing $\mathbf{Q}(t)=\mathbf{A}(t)-\mathbf{D}(t)$, it follows that this is at most

$$
\left(K_{Q}+K_{A}\right)|\mathcal{J}| \log \sigma_{\max }-\sum_{\substack{j \in \mathcal{J}: \\ Q_{j}(t)>0}} \frac{D_{j}(t+h)-D_{j}(t)}{h} \log D_{j}^{\prime}(t+h) .
$$

By Lemma 5, $D_{j}^{\prime}(t+h)$ is continuous at $h=0$ when $Q_{j}(t)>0$. Taking the limsup of the left hand side of (45), it follows from the above inequalities that

$$
D^{+} L(t) \leq\left(K_{Q}+K_{A}\right)|\mathcal{J}| \log \sigma_{\max }-\sum_{\substack{j \in \mathcal{J}: \\ Q_{j}(t)>0}} D_{j}^{\prime}(t) \log D_{j}^{\prime}(t) \leq \kappa_{L}
$$

for some constant $\kappa_{L}$, since $x \log x$ is bounded from below. It then follows from standard results on Dini derivatives that

$$
L(t)-L(s) \leq \kappa_{L}(t-s)
$$

(see, for instance, Theorem 3.4.5 on page 65 of Kannan and Krueger (1996)), which is the desired inequality.

iii) For $n \in \mathbb{N}$ and fixed $s, t$, and $u$, with $u \in[s, t]$, define the dyadic floor and ceiling $\lfloor u\rfloor_{n}$ and $\lceil u\rceil_{n}$ of $u$ by

$$
\begin{aligned}
& \lfloor u\rfloor_{n}:=\max _{k \in \mathbb{Z}_{+}}\left\{k(t-s) 2^{-n}+s: k(t-s) 2^{-n}+s \leq u\right\}, \\
& \lceil u\rceil_{n}:=\min _{k \in \mathbb{Z}_{+}}\left\{k(t-s) 2^{-n}+s: k(t-s) 2^{-n}+s>u\right\} ;
\end{aligned}
$$

note that $\lceil u\rceil_{n}-\lfloor u\rfloor_{n}=(t-s) 2^{-n}$. By interpolating terms and rewriting them in integral form, one has

$$
\begin{aligned}
L(t)-L(s) & =\sum_{k=1}^{2^{n}}\left(L\left(k(t-s) 2^{-n}+s\right)-L\left((k-1)(t-s) 2^{-n}+s\right)\right) \\
& =\int_{s}^{t} \frac{L\left(\lceil u\rceil_{n}\right)-L\left(\lfloor u\rfloor_{n}\right)}{\lceil u\rceil_{n}-\lfloor u\rfloor_{n}} d u=\lim _{n \rightarrow \infty} \int_{s}^{t} \frac{L\left(\lceil u\rceil_{n}\right)-L\left(\lfloor u\rfloor_{n}\right)}{\lceil u\rceil_{n}-\lfloor u\rfloor_{n}} d u \\
& \leq \int_{s}^{t} \limsup _{n \rightarrow \infty} \frac{L\left(\lceil u\rceil_{n}\right)-L\left(\lfloor u\rfloor_{n}\right)}{\lceil u\rceil_{n}-\lfloor u\rfloor_{n}} d u=\int_{s}^{t} L^{\prime}(u) d u
\end{aligned}
$$


The limit in the third equality holds trivially since the expression is not a function of $n$; the inequality follows from the Fatou Inequality since the integrand is bounded above by (28); and the last equality follows by using (27).

\section{Appendix B: Stability Results from Section 4.3}

For the proof of Lemma 3, we require the following technical lemma.

Lemma 8. For any $\boldsymbol{a} \in \mathcal{C}$, there exist $\epsilon>0$ and $c>0$, such that, for any $\mathbf{Q} \in \mathbb{R}_{+}^{|\mathcal{J}|}$ with $|\mathbf{Q}|>0$, one has $Q_{j} \geq c|\mathbf{Q}|$ and $\sigma_{j}(\mathbf{Q}) \geq(1+\epsilon) a_{j}$ for some queue $j$.

Proof Since the assertion is trivial if $a_{j}=0$ for some $j$, we can assume that $a_{j}>0$ for all $j$.

Since $\boldsymbol{a} \in \mathcal{C}$, there exists an $\epsilon>0$ such that $(1+\epsilon)^{2} \boldsymbol{a} \in \mathcal{C}$. Moreover, since $(1+\epsilon)^{2} \boldsymbol{a}$ is suboptimal,

$$
\sum_{j \in \mathcal{J}} Q_{j} \log \sigma_{j}(\mathbf{Q}) \geq \sum_{j \in \mathcal{J}} Q_{j} \log \left((1+\epsilon)^{2} a_{j}\right)
$$

hence

$$
\sum_{j \in \mathcal{J}} Q_{j} \log \frac{\sigma_{j}(\mathbf{Q})}{(1+\epsilon) a_{j}} \geq|\mathbf{Q}| \log (1+\epsilon)
$$

which implies the existence of at least one queue, say $j$, with

$$
Q_{j} \log \frac{\sigma_{j}(\mathbf{Q})}{(1+\epsilon) a_{j}} \geq \frac{|\mathbf{Q}|}{|\mathcal{J}|} \log (1+\epsilon)>0 .
$$

This implies that $\sigma_{j}(\mathbf{Q})>(1+\epsilon) a_{j}$, as the logarithm on the right hand side is positive, and also that

$$
\frac{Q_{j}}{|\mathbf{Q}|} \geq \frac{1}{|\mathcal{J}|} \log (1+\epsilon)\left(\log \frac{\sigma_{j}(\mathbf{Q})}{(1+\epsilon) a_{j}}\right)^{-1} \geq \frac{1}{|\mathcal{J}|} \log (1+\epsilon)\left(\log \frac{\sigma_{\max }}{(1+\epsilon) a_{\min }}\right)^{-1},
$$

where $a_{\min }:=\min \left\{a_{j}: j \in \mathcal{J}\right\}$. Denoting by $c>0$ the right hand side of this expression, the desired lower bound on $Q_{j}$ follows.

We now prove Lemma 3 , which gives a lower bound on the amount $Q_{j}(t)$ will change over time for some $j$. The main idea is that, if $Q_{j}(t)$ remains nearly constant, then, using Lemma 8 , it will follow that one of the queues $j$ will empty at a faster rate than mass enters its route, which will provide a contradiction over a long enough time interval.

Proof of Lemma 3 For $|\mathbf{Q}(0)|>0$, it follows from Lemma 8 that, for some queue $j, \sigma_{j}(\mathbf{Q}(0)) \geq$ $(1+\epsilon) a_{j}$, where $\boldsymbol{a}$ is the arrival rate vector. Denote by $\tilde{Q}_{j}(0)$ the quantity of packets at queue $j$ at time 0 , together with the packets already in the network then that will eventually be routed through $j$. Setting $\tilde{T}=2 \tilde{Q}_{j}(0) /\left(\epsilon a_{j}\right)$ and $T=2|\mathbf{Q}(0)| /\left(\epsilon a_{\min }\right)$ (with $a_{\min }:=\min _{j^{\prime} \in \mathcal{J}} a_{j^{\prime}}$ ), one has $T \geq \tilde{T} ;$ note that $a_{\min }>0$.

It is not possible that

$$
\sigma_{j}(\mathbf{Q}(t))>a_{j}\left(1+\frac{\epsilon}{2}\right) \quad \text { for all } t \in[0, T]
$$


For, if 48 were to hold, then $\int_{0}^{T} \sigma_{j}(\mathbf{Q}(t)) d t>a_{j}\left(1+\frac{\epsilon}{2}\right) T$, from which the contradiction

$$
0 \leq \tilde{Q}_{j}(T)=\tilde{Q}_{j}(0)+a_{j} T-\int_{0}^{T} \sigma_{j}(\mathbf{Q}(t)) d t<\tilde{Q}_{j}(0)-a_{j} \frac{\epsilon}{2} T \leq \tilde{Q}_{j}(0)-a_{j} \frac{\epsilon}{2} \tilde{T}=0
$$

would follow. Hence, for some $t \in[0, T], \sigma_{j}(\mathbf{Q}(t)) \leq a_{j}\left(1+\frac{\epsilon}{2}\right)$; since the index $j$ was obtained from Lemma 8, this implies that

$$
\sigma_{j}(\mathbf{Q}(0))-\sigma_{j}(\mathbf{Q}(t)) \geq a_{j} \frac{\epsilon}{2}
$$

By Lemma 5, there exists $\delta>0$ such that, for any $\mathbf{Q}$ with $|\mathbf{Q}-\mathbf{Q}(0)| \leq \delta|\mathbf{Q}(0)|$,

$$
\left|\sigma_{j}(\mathbf{Q})-\sigma_{j}(\mathbf{Q}(0))\right| \leq a_{j} \frac{\epsilon}{4}
$$

It therefore follows from 49 that, with possibly a new choice of $j$,

$$
\left|Q_{j}(t)-Q_{j}(0)\right|>\frac{\delta}{|\mathcal{J}|}|\mathbf{Q}(0)|
$$

We still need to show that the bound in $(33)$ is uniform in $|\mathbf{Q}(0)|>0$. The choice of $\epsilon$, which is determined by Lemma 8, does not depend on $\mathbf{Q}(0)$. Employing Lemma 5 and the compactness of $\left\{\mathbf{Q} \in \mathbb{R}_{+}^{|\mathcal{J}|}:|\mathbf{Q}|=1\right\}, \delta$ in $(51)$ can also be chosen so as not to depend on $\mathbf{Q}(0)$ since $Q_{j}(0) / \mathbf{Q}(0) \geq$ $c>0$ by Lemma 8, for $j$ in 50 . Setting $\kappa=\delta /|\mathcal{J}|$, we obtain 33 .

The elementary Lemma 4 follows from a straightforward Taylor expansion.

Proof of Lemma 4 Setting $F(z)=z \log z-(z-1)$, one has $F(1)=F^{\prime}(1)=0$ and $F^{\prime \prime}(z)=z^{-1}$. Expanding around 1 gives

$$
F(z)=\frac{1}{2} F^{\prime \prime}(\theta)(z-1)^{2}=\frac{1}{2 \theta}(z-1)^{2} \geq \frac{1}{2 \max (1, z)}(z-1)^{2}
$$

for some $\theta$ between 1 and $z$. Since

$$
x F(y / x)=y \log \left(\frac{y}{x}\right)-(y-x)
$$

for $x, y>0$, the desired inequality follows from

$$
x F(y / x) \geq \frac{x}{2 \max (1, y / x)}\left(\frac{y}{x}-1\right)^{2}=\frac{1}{2 \max (x, y)}(y-x)^{2} \geq \frac{1}{2 K}(y-x)^{2}
$$

for $K \geq \max (x, y)$. 


\section{Appendix C: Fluid Limit}

In Section 4, we employed Proposition 3 to conclude that the positive recurrence of a proportional switched network follows from the stability of the corresponding proportional switch fluid model. One of the main steps in showing Proposition 3 is Proposition 4 , which states that the proportional switch fluid model in Section 4.1 is the limit of scaled discrete time switched networks. We demonstrate Proposition 4 in this subsection; our approach is similar to the standard fluid limit approaches in Dai (1995) and Bramson (2008).

We consider a family of proportional switched networks indexed by $c \in \mathbb{Z}_{+}$, employing analogous notation to that introduced in Section 2, e.g., $A_{x}^{c}, D_{x}^{c}, Q_{x}^{c}$ for $x \in \mathcal{J} \cup \mathcal{K} \cup \mathcal{R}$. We will assume that the sum of the initial queue sizes of the queueing network is equal to $c$, that is, $c=\left|\mathbf{Q}^{c}(0)\right|=\sum_{j \in \mathcal{J}} Q_{j}^{c}(0)$. To simplify the proof, we couple the processes on the same probability space, assuming that the external arrival processes are identical for different $c$, that is, for $c \in \mathbb{Z}_{+}$and $r \in \mathcal{R}$,

$$
A_{r}^{c}(t)=A_{r}(t)
$$

For $x \in \mathcal{J} \cup \mathcal{K} \cup \mathcal{R}$ and $k \in \mathcal{K}$, we introduce the scaled processes $\bar{A}_{x}^{c}(t), \bar{D}_{x}^{c}(t), \bar{Q}_{x}^{c}(t)$, and $\bar{\Gamma}_{x}^{c}(t)$ on $t \geq 0$, by setting

$$
\bar{A}_{x}^{c}(t)=\frac{A_{x}^{c}(c t)}{c}, \bar{D}_{x}^{c}(t)=\frac{D_{x}^{c}(c t)}{c}, \bar{Q}_{x}^{c}(t)=\frac{Q_{x}^{c}(c t)}{c}, \bar{\Gamma}_{k}^{c}(t)=\frac{\Gamma_{k}^{c}(c t)}{c}
$$

for $t \in\left\{0, c^{-1}, 2 c^{-1}, 3 c^{-1}, \ldots\right\}$, and interpolating linearly. The following limit result holds for these processes. Here, $G=G_{1} \cap G_{2}$ is a set with $\mathbb{P}(G)=1$, where $G_{1}$ and $G_{2}$ will be specified shortly.

Proposition 4. For each $\omega \in G$, any scaled subsequence $\left(\bar{A}_{x}^{c_{i}}, \bar{D}_{x}^{c_{i}}, \bar{Q}_{x}^{c_{i}}, \bar{\Gamma}_{k}^{c_{i}}: x \in \mathcal{J} \cup \mathcal{K} \cup \mathcal{R}, k \in\right.$ $\left.\mathcal{K}, c_{1}<c_{2}<\ldots\right)$ of a sequence of proportional switched networks contains a further subsequence that converges uniformly on compact time intervals. Moreover, any such limit satisfies the proportional switch fluid model equations (3,8) and (17,19).

In order to demonstrate Proposition 4, we recall that, for each $r \in \mathcal{R},\left(A_{r}(t)-A_{r}(t-1): t \in \mathbb{Z}_{+}\right)$ is a collection of i.i.d. random variables with mean $a_{r}<\infty$. It thus follows from the Strong Law of Large Numbers that, on a set $G_{1}$ with $\mathbb{P}\left(G_{1}\right)=1$, one has that, for each $r \in \mathcal{R}, \bar{A}_{r}^{c}(t) \rightarrow a_{r} t$ as $c \rightarrow \infty$ uniformly on compact time intervals.

We also introduce, for each $j$, the martingales

$$
M_{j}^{c}(t)=\sum_{\tau=1}^{t}\left[\pi_{j}\left(\mathbf{Q}^{c}(\tau)\right)-\sigma_{j}\left(\mathbf{Q}^{c}(\tau)\right)\right],
$$

where $\pi_{j}(\cdot)$ and $\sigma_{j}(\cdot)$ are as in $(11)$, and set $\bar{M}_{j}^{c}(t)=M_{j}^{c}(c t) / c$. Since the increments of $\bar{M}_{j}^{c}(t)$ are uniformly bounded, it will follow from standard martingale bounds that, on a set with probability $1, \bar{M}_{j}^{c}(t) \rightarrow 0$ as $c \rightarrow \infty$, uniformly on compact time intervals for each $j \in \mathcal{J}$ : 
Lemma 9. On a set $G_{2}$ with $\mathbb{P}\left(G_{2}\right)=1$,

$$
\sup _{t \leq T}\left|\bar{M}_{j}^{c}(t)\right| \underset{c \rightarrow \infty}{\longrightarrow} 0
$$

for all $j \in \mathcal{J}$ and $T>0$.

We will demonstrate Lemma 9 after the proof of Proposition 4 .

The proof of Proposition 4 is relatively straightforward. Most of the work consists of showing the above limits satisfy the equations $(3,8)$ and $(17, \sqrt{19})$; the invariance of the equations $(3,8)$ under fluid scaling is an important ingredient.

Proof of Proposition 4. By the Arzelà-Ascoli Theorem (cf. Billingsley (1999)), any sequence of equicontinuous functions $X^{c_{i}}(t)$ on $[0, T], T \in(0, \infty)$, such that $\sup _{c_{i}}\left|X^{c_{i}}(0)\right|<\infty$, has a converging subsequence with respect to the uniform norm. In order to apply the theorem, we will show that the sequences $\left(\bar{A}_{x}^{c_{i}}, \bar{D}_{x}^{c_{i}}, \bar{Q}_{x}^{c_{i}}, \bar{\Gamma}_{k}^{c_{i}}\right)$ of 4-tuples satisfy both properties for $\omega \in G_{1}$.

Since $\left|\overline{\mathbf{Q}}^{c_{i}}(0)\right|=1$ and the other variables are initially 0 , the second property clearly holds. In order to show equicontinuity, we first note that each packet requires one unit of service, and so $\bar{D}_{x}^{c}(t)$ is Lipschitz for $x \in \mathcal{J} \cup \mathcal{R} \cup \mathcal{K}, k \in \mathcal{K}$; by equation (4), so is $\bar{\Gamma}_{k}^{c}(t)$. Hence, both variables are equicontinuous.

On the other hand, equicontinuity of $\bar{A}_{r}^{c_{i}}(t)$ follows by applying the Functional Strong Law of Large Numbers to $\bar{A}_{r}^{c_{i}}(t)$ for increasingly fine meshes in $t$. Equicontinuity for the remaining variables $\bar{A}_{x}^{c_{i}}(t)$ and $\bar{Q}_{x}^{c_{i}}(t)$ follows from straightforward calculations using the equations (38). Consequently, by the Azelà-Ascoli Theorem, every subsequence $\left\{\left(\bar{A}_{x}^{c_{i}}, \bar{D}_{x}^{c_{i}}, \bar{Q}_{x}^{c_{i}}, \bar{\Gamma}_{k}^{c_{i}}: x \in \mathcal{J} \cup \mathcal{K} \cup \mathcal{R}, k \in \mathcal{K}\right)\right\}$ has a further subsequence that converges uniformly on $[0, T]$.

We now show that equations (3, 8 ) and (17, 19) hold for the limit of any converging subsequence of $\left\{\left(\bar{A}_{x}^{c_{i}}, \bar{D}_{x}^{c_{i}}, \bar{Q}_{x}^{c_{i}}, \bar{\Gamma}_{k}^{c_{i}}: x \in \mathcal{J} \cup \mathcal{K} \cup \mathcal{R}, k \in \mathcal{K}\right)\right\}$; we will demonstrate this on $G_{1}$ for all equations except (18, 19). With the exception of (65), equations (3-8) clearly hold for any limit since each holds under the prelimit. We must take a little more care for expressions (66), because some minor error is introduced by linear interpolating terms; the bound

$$
\left|D_{k}^{c}(s)-\Gamma_{k}^{c}\left(D_{j}^{c}(s)\right)\right| \leq\left|D_{k}^{c}(\lceil s\rceil)-D_{k}^{c}(\lfloor s\rfloor)\right| \leq \sigma_{\max }
$$

shows that this error vanishes in the limit. On $G_{1}, 17$ follows directly from the Strong Law of Large Numbers.

We still need to demonstrate $(18)$ for $\sigma(\mathbf{Q})$ satisfying $(19)$, which we show for $\omega \in G_{2}$, where $G_{2}$ will be chosen as in Lemma 9. Let $\left(Q_{j}, D_{j}: j \in \mathcal{J}\right)$ be the limit of a subsequence of $\left\{\left(\bar{Q}_{j}^{c_{i}}, \bar{D}_{j}^{c_{i}}: j \in\right.\right.$ $\mathcal{J})\}$. Suppose that $Q_{j}(t)>\epsilon$ for some $\epsilon>0$ and a given $j$. Then, by the continuity of $\mathbf{Q}(t)$, there exists $\delta>0$ such that $Q_{j}(t+h)>\epsilon$ for all $h$ satisfying $|h| \leq \delta$. Since convergence is uniform, there also exists $c^{\prime}$ such that, for all $c_{i} \geq c^{\prime}, \bar{Q}_{j}^{c_{i}}(t+h)>\epsilon$ for all such $h$. 
For $u \in \mathbb{R}_{+}$, denote by $\lfloor u\rfloor_{c_{i}}$ be the largest value of $k c_{i}^{-1}, k \in \mathbb{Z}$, that is at most $u$. The scaled departures over $\left(\lfloor t\rfloor_{c_{i}},\lfloor t+h\rfloor_{c_{i}}\right]$ can be rewritten as

$$
\begin{aligned}
& \bar{D}_{j}^{c_{i}}\left(\lfloor t+h\rfloor_{c_{i}}\right)-\bar{D}_{j}^{c_{i}}\left(\lfloor t\rfloor_{c_{i}}\right)=\frac{1}{c_{i}} \sum_{\tau=\lfloor t\rfloor_{c_{i}}+1 / c_{i}}^{\lfloor t+h\rfloor_{c_{i}}} \pi_{j}\left(\overline{\mathbf{Q}}^{c_{i}}(\tau)\right) \\
& =\frac{1}{c_{i}} \sum_{\tau=\lfloor t\rfloor_{i}+1 / c_{i}}^{\lfloor t+h\rfloor_{c_{i}}} \sigma_{j}\left(\overline{\mathbf{Q}}^{c_{i}}(\tau)\right)+\bar{M}_{j}^{c_{i}}\left(\lfloor t+h\rfloor_{c_{i}}\right)-\bar{M}_{j}^{c_{i}}\left(\lfloor t\rfloor_{c_{i}}\right) \\
& =\int_{\lfloor t\rfloor_{c_{i}}+1 / c_{i}}^{\lfloor t+h\rfloor_{c_{i}}} \sigma_{j}\left(\overline{\mathbf{Q}}^{c_{i}}\left(\lfloor\tau\rfloor_{c_{i}}\right)\right) d \tau+\bar{M}_{j}^{c_{i}}\left(\lfloor t+h\rfloor_{c_{i}}\right)-\bar{M}_{j}^{c_{i}}\left(\lfloor t\rfloor_{c_{i}}\right),
\end{aligned}
$$

where the summations are understood to be over values in $\left\{0, c_{i}^{-1}, 2 c_{i}^{-1}, \ldots\right\}$. The square bracketed terms on the last line of (53) are bounded. For $\omega \in G_{2}$, with $G_{2}$ as in Lemma 9 , the last two terms in (53) converge uniformly to zero. On the other hand, by uniform convergence, the terms $\overline{\mathbf{Q}}^{c_{i}}\left(\lfloor\tau\rfloor_{c_{i}}\right)$ on the subsequence converge to $\mathbf{Q}_{j}(\tau)$. Also, $\sigma_{j}(\mathbf{Q})$ is bounded and continuous on $Q_{j}>0$. Thus, applying the Bounded Convergence Theorem, it follows from (53) that

$$
D_{j}(t+h)-D_{j}(t)=\int_{t}^{t+h} \sigma_{j}(\mathbf{Q}(\tau)) d \tau
$$

where $\sigma_{j}$ is as in (19). Dividing by $h$ and taking the limit $h \searrow 0$ implies $D^{\prime}{ }_{j}(t)=\sigma_{j}(\mathbf{Q}(t))$, and hence that (18) also holds.

Proof of Lemma 9 For $\epsilon>0$, let $\tau_{\epsilon}$ denote the stopping time for the event $\left\{\left|M_{j}^{c}(t)\right| \geq c \epsilon\right\}$. Since, for each $c, M_{j}^{c}$ is a martingale with increments uniformly bounded by $\sigma_{\max }$, it follows by applying the Azuma-Hoeffding Inequality (Williams 1991, E14.2) to $\left\{M_{j}^{c}\left(\min \left(\tau_{\epsilon}, s\right)\right)\right\}_{s \leq c T}$ that

$$
\mathbb{P}\left(\max _{t: t \leq c T}\left|M_{j}^{c}(t)\right| \geq c \epsilon\right)=\mathbb{P}\left(\left|M_{j}^{c}\left(\min \left(\tau_{\epsilon}, c T\right)\right)\right| \geq c \epsilon\right) \leq 2 \exp \left\{-\frac{c^{2} \epsilon^{2}}{2 c T \sigma_{\max }^{2}}\right\} .
$$

After summing over $c \in \mathbb{N}$, the right hand side of the above inequality is finite and hence, by the Borel-Cantelli Lemma,

$$
\limsup _{c \rightarrow \infty} \max _{t: t \leq c T} \frac{1}{c}\left|M_{j}^{c}(t)\right| \leq \epsilon
$$

holds almost surely. Since $\epsilon>0$ is arbitrary, this implies the claim in the lemma.

\section{Appendix D: Positive Recurrence}

In this subsection, we demonstrate Proposition 3, which states that positive recurrence for a FIFO proportional switched network follows from the fluid model stability of the corresponding proportional switch fluid model. The main tools are Proposition 4 of the previous subsection and the Multiplicative Foster's Criterion. 
Bramson, D'Auria and Walton: Proportional Switching

Article submitted to Operations Research; manuscript no. (Please, provide the manuscript number!)

Proof of Proposition 3 One can check that, for each $t \geq 0$, the sequence of queue sizes $\left\{\left|\overline{\mathbf{Q}}^{c}(t)\right|\right\}_{c}$ of the scaled proportional switched networks in Proposition 4 is uniformly integrable. This follows quickly from the inequality

$$
\left|\overline{\mathbf{Q}}^{c}(t)\right|=\sum_{j \in \mathcal{J}} \bar{Q}_{j}^{c}(t) \leq \sum_{j \in \mathcal{J}} \frac{Q_{j}^{c}(0)}{c}+\sum_{r \in \mathcal{R}} \frac{A_{r}^{c}(t)}{c}
$$

since $A_{r}^{c}(t)$ is a sum of i.i.d. random variables with finite mean (see, e.g., Bramson (2008, Lemma $4.13,(4.81)))$.

On the other hand, by Proposition 4 , on a set of probability one, every subsequence of $\overline{\mathbf{Q}}^{c}(t)$ has a further subsequence that converges uniformly on compact time intervals to a fluid model solution $\mathbf{Q}(t)$ of $(3 \sqrt{8})$ and $(17 \sqrt{19})$, with $|\mathbf{Q}(0)|=1$. By Theorem 2 , this fluid model is stable; hence all fluid model solutions with $|\mathbf{Q}(0)|=1$ satisfy $|\mathbf{Q}(t)|=0$ for $t \geq \gamma$, with $\gamma$ not depending on the particular fluid model solution.

It follows from this that, on a set of probability one, every subsequence of $\left|\overline{\mathbf{Q}}^{c}(\gamma)\right|$ has a further subsequence converging to zero; consequently, $\left|\overline{\mathbf{Q}}^{c}(\gamma)\right|$ also converges to zero on a set of probability one. By the above uniform integrability and almost sure convergence of $\left|\overline{\mathbf{Q}}^{c}(\gamma)\right|$, it follows that

$$
\lim _{c \rightarrow \infty} \mathbb{E}\left|\overline{\mathbf{Q}}^{c}(\gamma)\right|=0,
$$

and so, if $c \geq \tilde{c}$ for appropriate $\tilde{c}$, then $\mathbb{E}\left|\overline{\mathbf{Q}}^{c}(\gamma)\right| \leq 1 / 2$.

The last inequality is equivalent to $\mathbb{E}\left|\mathbf{Q}^{c}(\gamma c)\right| \leq c / 2$; together with (54), it implies that, for large enough $\kappa$ and all $c$,

$$
\mathbb{E}\left|\mathbf{Q}^{c}(\gamma(\max (c, \kappa)))\right| \leq \max (c, \kappa) / 2 .
$$

The inequality (55) satisfies the main premise of the Multiplicative Foster's Criterion (Bramson 2008, Proposition 4.6, (4.28)), and therefore implies the positive recurrence of the corresponding proportional switched network, which completes the proof of the proposition. (The criterion is stated in Bramson (2008) for continuous time Markov processes; however, both the criterion and its proof carry over to discrete time. Alternatively, the discrete time Markov process can be embedded in continous time. The petite set assumption in the criterion is automatically satisfied in our framework since the empty state will be hit with uniformly high probability from sets with a bounded number of packets.)

\section{Acknowledgments}

The research of the first author was partially supported by NSF grants DMS-1105668 and DMS-1203201. The research of the second author was partially supported by the Spanish Ministry of Economy and Competitiveness Grants MTM2013-42104-P via FEDER funds; he thanks the ICMAT (Madrid, Spain) Research Institute that kindly hosted him while developing this project. The research of the third author was funded by the VENI research programme, which is financed by the Netherlands Organisation for Scientific Research (NWO). 